OPEN ACCESS

Edited by:

Guohui Liu,

Huazhong University of Science and

Technology, China

Reviewed by:

Zhong Wu,

Tongji University, China

Min Nie,

Cornell University, United States

*Correspondence:

Chao Chen

chenchao027@163.com

Jingfeng $L i$

jingfengli@whu.edu.cn

${ }^{+}$These authors have contributed equally to this work

Specialty section: This article was submitted to

Stem Cell Research,

a section of the journal

Frontiers in Cell and Developmental

Biology

Received: 06 October 2021

Accepted: 26 October 2021

Published: 09 November 2021

Citation:

Hao Z, XU Z, Wang X, Wang Y, Li H,

Chen T, Hu Y, Chen R, Huang $K$,

Chen C and Li J (2021) Biophysical

Stimuli as the Fourth Pillar of Bone

Tissue Engineering.

Front. Cell Dev. Biol. 9:790050.

doi: 10.3389/fcell.2021.790050

\section{Biophysical Stimuli as the Fourth Pillar of Bone Tissue Engineering}

\author{
Zhuowen $\mathrm{Hao}^{1 \dagger}$, Zhenhua $\mathrm{Xu}^{1+}$, Xuan Wang ${ }^{1 \dagger}$, Yi Wang ${ }^{1}$, Hanke $\mathrm{Li}^{1}$, Tianhong Chen ${ }^{1}$, \\ Yingkun Hu${ }^{1}$, Renxin Chen ${ }^{1}$, Kegang Huang ${ }^{2}$, Chao Chen ${ }^{3,4 *}$ and Jingfeng $\mathrm{Li}^{{ }^{1 *}}$ \\ ${ }^{1}$ Department of Orthopedics, Zhongnan Hospital of Wuhan University, Wuhan, China, ${ }^{2}$ Wuhan Institute of Proactive Health \\ Management Science, Wuhan, China, ${ }^{3}$ Department of Orthopedics, Union Hospital, Tongji Medical College, Huazhong University \\ of Science and Technology, Wuhan, China, ${ }^{4}$ Department of Orthopedics, Hefeng Central Hospital, Enshi, China
}

The repair of critical bone defects remains challenging worldwide. Three canonical pillars (biomaterial scaffolds, bioactive molecules, and stem cells) of bone tissue engineering have been widely used for bone regeneration in separate or combined strategies, but the delivery of bioactive molecules has several obvious drawbacks. Biophysical stimuli have great potential to become the fourth pillar of bone tissue engineering, which can be categorized into three groups depending on their physical properties: internal structural stimuli, external mechanical stimuli, and electromagnetic stimuli. In this review, distinctive biophysical stimuli coupled with their osteoinductive windows or parameters are initially presented to induce the osteogenesis of mesenchymal stem cells (MSCs). Then, osteoinductive mechanisms of biophysical transduction (a combination of mechanotransduction and electrocoupling) are reviewed to direct the osteogenic differentiation of MSCs. These mechanisms include biophysical sensing, transmission, and regulation. Furthermore, distinctive application strategies of biophysical stimuli are presented for bone tissue engineering, including predesigned biomaterials, tissueengineered bone grafts, and postoperative biophysical stimuli loading strategies. Finally, ongoing challenges and future perspectives are discussed.

Keywords: biophysical stimuli, mesenchymal stem cells, osteoinductive mechanisms, biophysical transduction, osteogenesis

\section{INTRODUCTION}

After trauma, bone tissue shows self-healing property, but this ability is limited for critical bone defects (average diameter over $2 \mathrm{~cm}$ in humans) caused by serious injury, tumor excision, or other orthopedic diseases (Lopes et al., 2018). Bone healing failure, which occurs in 5-10\% of all patients with bone fracture, generally causes delayed union (healing process over 3 months) or non-union (healing process over 9 months without obvious bone regeneration in the first 3 months) (Zura et al., 2016; Wojda and Donahue, 2018). Autologous bone grafting is currently the gold standard for the healing of critical bone defects because it provides three critical components: an osteoconductive substrate, osteoinducive signals, and preosteoblastic cells (Yong et al., 2020). However, the strategy fails to meet clinical requirements because of limited autografts, potential donor site complications (such as infections, chronic pain, and bleeding), and the risk of graft failure (Roseti et al., 2017; Yang et al., 2018).

Bone tissue engineering has been becoming an ideal strategy to replace autologous bone grafting, and it is composed of three pillars to emulate the basic components of autografts: biomaterial scaffolds, bioactive molecules, and stem cells (Huang et al., 2020). Bioactive molecules are generally 


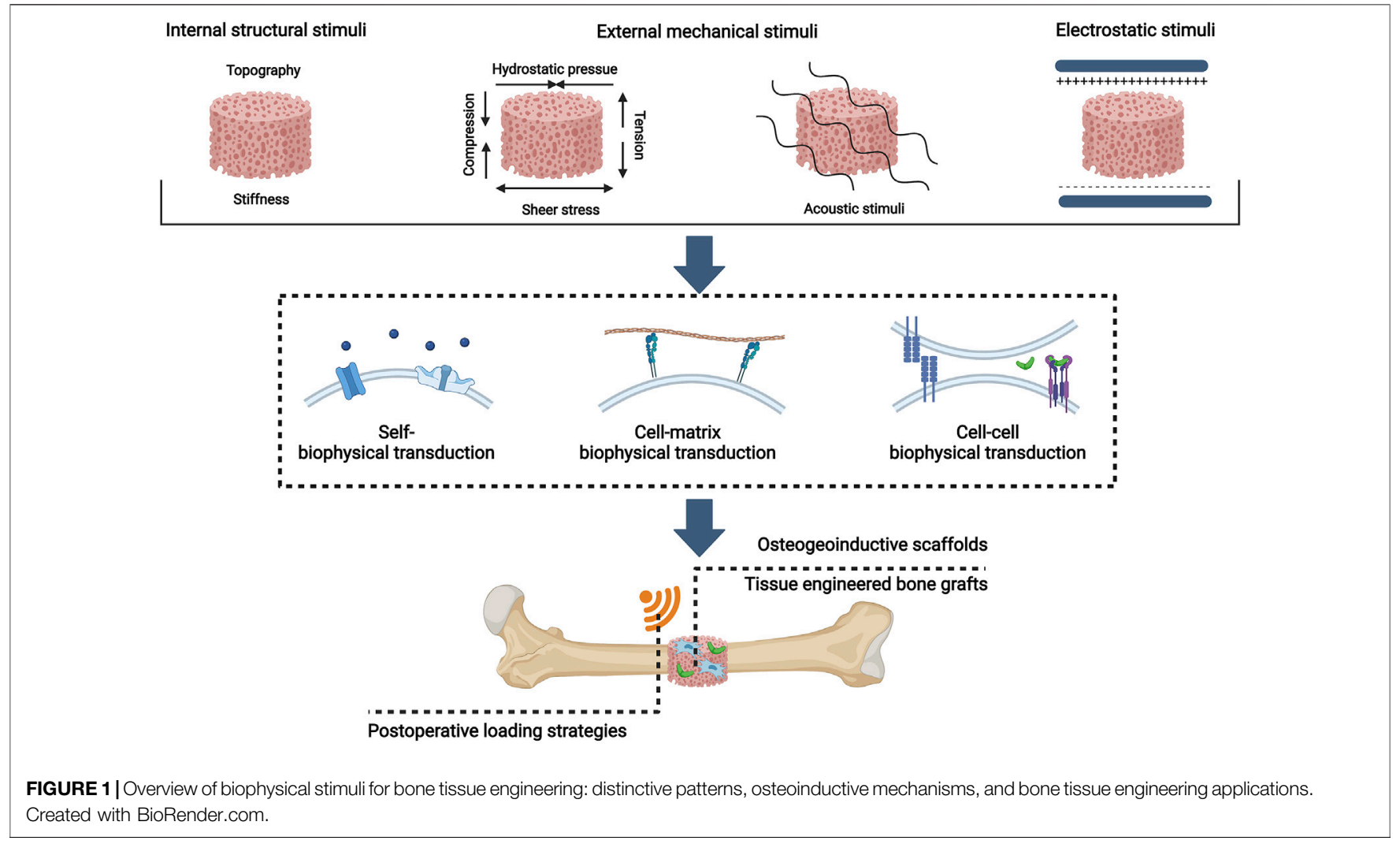

in the form of recombinant growth factors or small molecular bioactive peptides to provide osteoinductive properties (Yang et al., 2018). But the delivery of bioactive molecules shows some limitations: 1) initial burst release, 2) declined biological activity, 3) high therapeutic dosage, and 4) potential side effects (Krishnan et al., 2017; Bertrand et al., 2020). Therefore, another pillar showing osteoinductive properties needs to be incorporated into bone tissue engineering for bone regeneration or bone healing.

Biophysical stimuli have attracted great attention for bone regeneration because of their great promise as the fourth pillar of bone tissue engineering. From Wolff's law to Frost's "mechanostat theory", a plethora of evidence verifies that bone is a mechanosensitive tissue (Tyrovola 2015; Haffner-Luntzer et al., 2016; Qin E. C. et al., 2020). Multiple bone cells that respond to biophysical stimuli include osteocytes, osteoblasts, osteoclasts, bone lining cells, and mesenchymal stem cells (MSCs) (Steward and Kelly, 2015; Stewart et al., 2020). In bone tissue engineering, the osteogenic differentiation of MSCs is the most important process. Therefore, this review focuses on the osteoinductive effects of biophysical stimuli toward MSCs. Biophysical stimuli with osteoinductive properties can be categorized into three groups depending on their physical properties: internal structural stimuli, external mechanical stimuli, and electromagnetic stimuli.

External mechanical stimuli were proposed as the fourth pillar of bone regeneration by Lopes et al. (2018). Here we suggest that the concept can cover even more comprehensive forms of biophysical stimuli. In this review, we first update the fourth pillar of bone tissue engineering as biophysical stimuli and summarize distinctive biophysical stimuli with their osteoinductive windows for MSC osteogenesis, including internal structural stimuli, external mechanical stimuli, and electromagnetic stimuli. Then, a novel concept of biophysical transduction (a process of sensing, transmission, and regulation) that incorporates mechanotransduction and electrocoupling is proposed to interpret the osteoinductive mechanisms of biophysical stimuli for the osteogenic differentiation of MSCs. And biophysical stimuli, depending on sensing mechanisms, can be divided into self-biophysical transduction, cell-matrix transduction, and cell-cell biophysical transduction. Moreover, the application strategies of biophysical stimuli as the fourth pillar of bone tissue engineering are presented, which include preconstructed scaffolds with osteoinductive properties, tissue engineered bone grafts (TEBGs), and postoperative biophysical stimuli loading strategies. (Figure 1). This review aims to propose a novel and comprehensive concept that biophysical stimuli show potential to be used as the fourth pillar of bone tissue engineering.

\section{DISTINCTIVE BIOPHYSICAL STIMULI FOR BONE TISSUE ENGINEERING}

MSCs can be obtained from various tissues, including bone marrow-derived MSCs (BMSCs), periosteum-derived stem cells (PDSCs), adipose-derived stem cells (ADSCs), and periodontal 

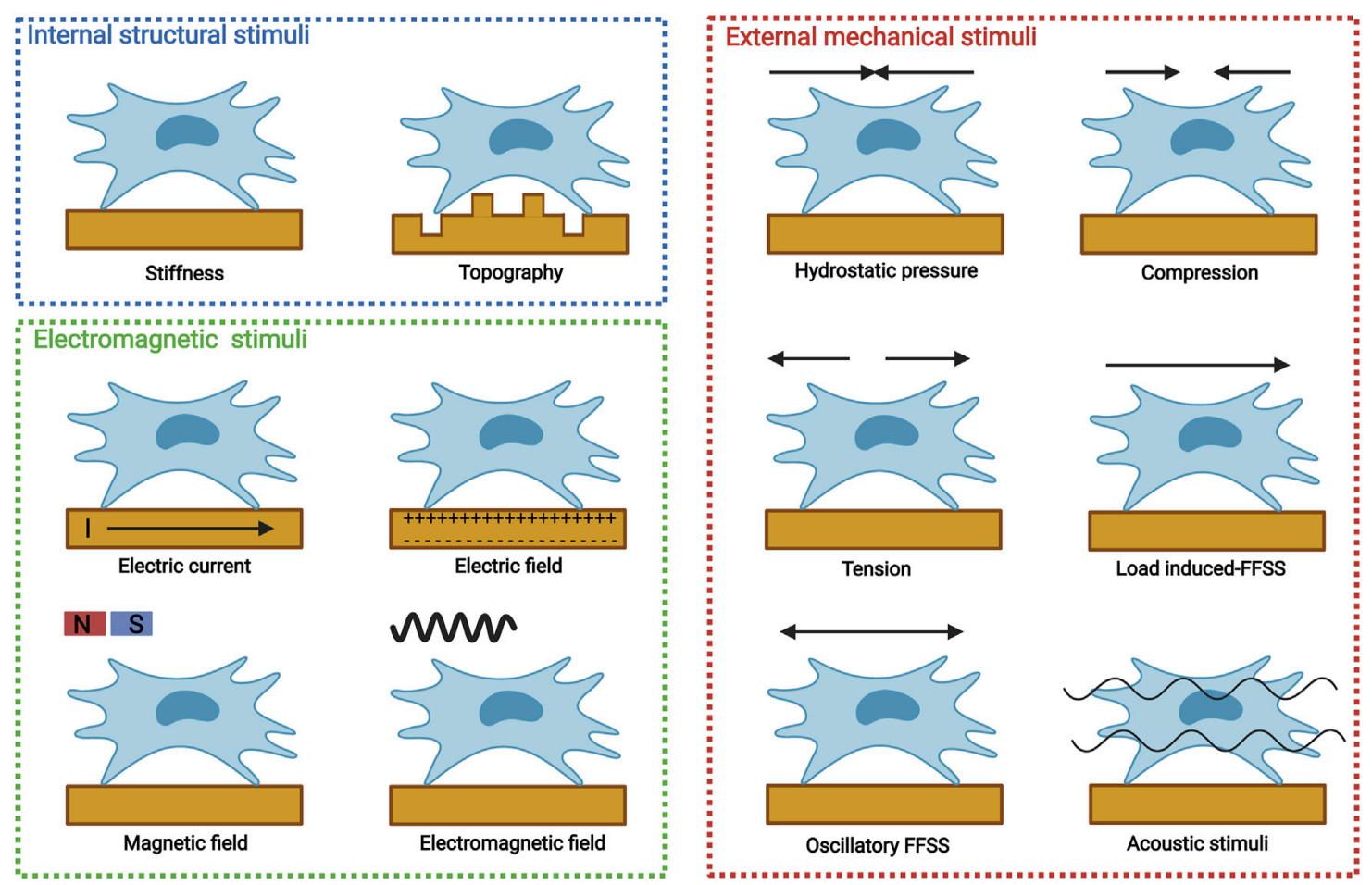

FIGURE 2 | Distinctive biophysical stimuli for bone tissue engineering, including internal structural stimuli (stiffness and topography), external mechanical stimuli [hydrostatic pressure, compression, tension, load induced-fluid flow shear stress (FFSS), oscillatory FFSS without load, and acoustic stimuli], and electromagnetic stimuli (electric current, electric field, magnetic field, and electromagnetic field). Created with BioRender.com.

ligament stem cells (PDLCs). For bone tissue engineering, MSCs are introduced to biomaterials either by direct encapsulation or indirect recruitment. Biophysical stimuli could modulate various MSC processes, including migration, proliferation, and differentiation. Distinctive biophysical stimuli with different parameters may result in different MSC specifications. Biophysical stimuli for osteogenic differentiation should be limited by one or several parameters, which can be termed as osteoinductive windows. Depending on physical properties, biophysical stimuli can be categorized into internal structural stimuli, eternally mechanical stimuli, and electromagnetic stimuli (Figure 2).

\subsection{Internal Structural Stimuli}

Internal structural stimuli are derived from matrix microenvironment where MSCs survive and grow. In human, distinctive tissues show different mechanical properties, among which matrix stiffness and topography determine the fate of MSCs.

\subsubsection{Matrix Stiffness}

Matrix stiffness is the rigidity or elasticity of the three dimensional (3D) microenvironment. MSCs show different cell shapes when loaded on the surface of collagens with distinctive stiffness (Engler et al., 2006). In specific microenvironment with different matrix stiffness, they transfer from the initially round shape to a branched $(0.1-1 \mathrm{kPa})$, spindle $(8-17 \mathrm{kPa})$, or polygonal
(25-40 kPa) shape, which then determines their commitment for neurogenic, myogenic, or osteogenic differentiation (Engler et al., 2006). The results can be also supported by the fact that spread, flattened, and adherent MSCs undergo osteogenic differentiation, whereas unspread and round MSCs undergo adipogenic differentiation (McBeath et al., 2004). However, in a 3D microenvironment, the influence of cell shape transfers to nanoscale integrin binding and the rearrangement of cell adhesion ligands, which could stimulate the osteogenic differentiation of MSCs by contractility (Huebsch et al., 2010). In addition, 3D matrix with a stiffness of $11-30 \mathrm{kPa}$ predominantly stimulates osteogenesis (Huebsch et al., 2010).

\subsubsection{Topography}

Topography is another mechanical property related to cell adhesion. According to the patterning size, topography exerts effects on different levels, including macroscale colony level $(>100 \mu \mathrm{m})$, microscale cell level $(0.1-100 \mu \mathrm{m})$, and nanoscale receptor level $(1.0-100 \mathrm{~nm})$, among which nanotopography influences the commitment of MSCs (Prè et al., 2013). Various nanopatterns (such as nanopits, nanorods, nanopillars, or nanocolumns) can be modified on the surface of biomaterials for the osteogenic differentiation of MSCs, and specific osteoinductive parameters (shape, diameter, spacing, height, or depth) vary greatly among these nanopatterns and different fabrication techniques (Dobbenga et al., 2016). However, all osteoinductive windows of different nanotopographies show 
nanoscale-controlled disorder, a nanopattern that is not completely random and not highly ordered (Prè et al., 2013). Dalby et al. first fabricated five nanotopographies on the surface of polymethylmethacrylate embossed with nanopits (diameter $120 \mathrm{~nm}$, depth $100 \mathrm{~nm}$ ): highly ordered hexagonal array (center-center spacing $300 \mathrm{~nm}$ ), highly ordered square array (center-center spacing $300 \mathrm{~nm}$ ), disordered square array with a controlled displacement of $20 \mathrm{~nm}$ (center-center spacing $300 \pm$ $20 \mathrm{~nm}$ ), and disordered square array with a controlled displacement of $50 \mathrm{~nm}$ (center-center spacing $300 \pm 50 \mathrm{~nm}$ ) (Dalby et al., 2007). All highly ordered groups and completely random groups fail to sufficiently induce the osteogenic differentiation of MSCs, but both disordered nanotopographies with controlled displacement show osteoinductive properties; those with a controlled displacement of $50 \mathrm{~nm}$ are superior to those with a controlled displacement of $20 \mathrm{~nm}$ in terms of osteocalcin (OCN), osteopontin (OPN), and bone nodule contents (Dalby et al., 2007). Zhang et al. utilized nanorods to explore the osteoinductive properties of nanotopography with controlled disorder (Zhou J. et al., 2016). They fabricated five nanopatterns with different interrod spacings (302.7 \pm 10.5 , $137.2 \pm 7.5,95.9 \pm 3.8,66.8 \pm 4.1$, and $32.6 \pm 2.7 \mathrm{~nm})$. And they found that the group with interrod spacings over $137 \mathrm{~nm}$ impedes MSC osteogenesis, the group with interrod spacings below $96 \mathrm{~nm}$ facilitates osteogenic differentiation, and the $66.8 \pm$ $4.1 \mathrm{~nm}$ group shows preferable osteoinduction (Zhou X. et al., 2016). The above results suggested that the interspacing of nanopattern determines the osteoinductive windows of nanotopography. And the modification of nonopits may need relatively large interspacing because the spacing area supports cell adhesion, whereas the modification of nanorods or nanopillars needs relatively small interspacing because they support cell adhesion. Although nanopillars with different heights (Sjöström et al., 2009; McNamara et al., 2011; Sjöström et al., 2013) or nanopits with distinctive diameters (Lavenus et al., 2011) show different osteoinductive properties, nanopattern interspacing changes with height. Thus, whether or not the height or diameter of nanopatterns truly controls the osteogenic differentiation of MSCs remains unknown, and further studies should make spacing constant while changing other parameters.

\subsection{External Mechanical Stimuli}

External mechanical stimuli are derived from external forces, which could exert effects on MSCs continuously or cyclically. External mechanical stimuli that show osteoinductive properties include hydrostatic pressure (HP), compression, tension, loadinduced fluid flow shear stress (FFSS), oscillatory FFSS, and acoustic stimuli.

\subsubsection{Hydrostatic Pressure}

MSCs reside in a fluid-filled microenvironment. Thus, HP affects the fate of MSCs, which may exert homogenous compression to MSCs. Huang et al. used cyclic HP $(0.5 \mathrm{MPa}, 0.5 \mathrm{~Hz})$ by a perfusion bioreactor and found that the sinusoidal profile could promote osteogenic differentiation, but the proliferation is spoiled (Huang and Ogawa, 2012). HP with high magnitude does not accord with physiological HP; hence, high-magnitude HP may be limited for regenerative medicine. From a physiological perspective, MSCs in the bone marrow are exposed to static intramedullary pressure (approximately $4 \mathrm{kPa}$ ), which increases to $50 \mathrm{kPa}$ when exposed to external mechanical stimuli, and stem cells in the perivascular space and Haversian channels may experience $300 \mathrm{kPa}$ pressure. Thus, researchers further compared the osteogenic effects of three HPs $(10,100$, and $300 \mathrm{kPa})$ with different frequencies $(0.5,1$, and $2 \mathrm{~Hz})$ and durations $(1,2$, and $4 \mathrm{~h})$ and found that $\mathrm{HP}$ with $300 \mathrm{kPa}$ and $2 \mathrm{~Hz}$ produces the most effective osteoinductive property (Stavenschi et al., 2018). However, the collagen synthesis and mineral deposition are similar among different groups, showing that $\mathrm{HP}$ with $10 \mathrm{kPa}$ is sufficient to induce MSC osteogenesis (Stavenschi et al., 2018). Reinwald et al. found that intermittent $\mathrm{HP}(270 \mathrm{kPa}, 1 \mathrm{~Hz}, 60 \mathrm{~min} /$ day, 21 days $)$ promotes the osteogenic differentiation of MSCs when loaded onto poly (E-caprolactone) (PCL) scaffolds (Reinwald and El Haj, 2018). Altogether, cyclic or intermittent HP with magnitude $10-300 \mathrm{kPa}$ induces MSC osteogenesis.

\subsubsection{Compression}

In addition to HP, compression (physiological strain from 0.2 to $0.4 \%)$ is induced on the vertical direction of the force when natural bone is compressed (Al Nazer et al., 2012). Depending on loading pattern, compression can be classified into uniaxial compression and equiaxial compression, both of which could induce the osteogenic differentiation of MSCs when they are limited by specific parameters. The stiffness of biomaterials is enhanced to promote osteogenesis when scaffolds are exposed to compression (Baumgartner et al., 2018). Among parameters describing compression, the magnitude of compression strain determines the fate of compressed MSCs. However, osteoinductive compression strain magnitude varies greatly because of different compression devices, durations, and biomaterials. For relatively high compression strain $(\geq 1.5 \%)$, whether or not high-magnitude compression stimulates osteogenesis remains controversial (Haudenschild et al., 2009; Sittichokechaiwut et al., 2010; Aziz et al., 2019; Schreivogel et al., 2019). It was revealed that $5 \%$ compression could induce the osteogenic differentiation of MSCs loaded onto polyurethane scaffolds without biochemical cues, which show comparable osteoinduction with dexamethasone (Sittichokechaiwut et al., 2010). However, Horner et al. adopted four compression strains $(5,10,15$, and 20\%) and found that osteogenic markers decrease and chondrogenic markers increase in a magnitude-dependent manner (Horner et al., 2018). Haudenschild et al. also found that $\pm 5 \%$ bulk strain with $5 \%$ offset stimulates chondrogenic differentiation (Haudenschild et al., 2009). Indeed, high compression strain inhibits the expression of Runt-related transcription factor 2 (RUNX2), but the expression of bone Morphogenetic Protein-2 (BMP-2) is interestingly upregulated (Schreivogel et al., 2019). Thus, one potential explanation for this discrepancy is that abundant culture medium blocks the effects of mechanosensitive autocrine factors, which impede osteogenic differentiation. Schreivogel et al. further explored the effects of autocrine 
factors by improving the number of scaffolds containing MSCs and reducing the volume of culture medium; results showed that 5 and $10 \%$ compression could promote the osteogenic differentiation of MSCs (Schreivogel et al., 2019). Therefore, high-magnitude compression may induce osteogenesis by mechanosensitive autocrine factors, such as BMP-2, and the ratio of cell number and medium volume may determine the fate of MSCs. Compared with high-magnitude compression, lowmagnitude compression could directly promote the expression of osteogenic markers. A previous study seeded MSCs to monetite calcium phosphate scaffolds and then subjected them to compression $(0.4 \%, 0.1 \mathrm{~Hz})$ (Gharibi et al., 2013). After $2 \mathrm{~h}$ stimulation, some immediate-early response genes are activated, which promote the expression of other genets (such as RUNX-2) to induce the proliferation and osteogenic differentiation of MSCs (Gharibi et al., 2013). Ravichandran et al. adopted low compression strains $(0.22,0.88$, and $1.1 \%)$ and found that the $0.22 \%$ group induces more alkaline phosphatase (ALP) and calcium than the other groups (Ravichandran et al., 2017). These studies indicate that although compression with high magnitude stimulates osteogenesis by mechanosensitive autocrine factors, the precise ratio of cell number to medium volume is difficult to control. Thus, physiological compression $(0.2-0.4 \%)$ shows great promise for MSC osteogenesis.

\subsubsection{Tension}

When natural bone is pulled, tension is also induced on the vertical direction of the force. Depending on the loading pattern, tension could be divided into uniaxial compression and equiaxial compression. Different from compression, tension with high strain (such as 5\%) promotes osteogenic differentiation and inhibits adipogenic differentiation (Li et al., 2015a). Tensile strain-inducing cell culture plates are generally used to study the effects of tension. In these two dimensional (2D) models, MSCs are initially seeded on the cell culture plates coated with the matrix, and then the plates are subjected to tension strain, which indirectly exerts tension to MSCs. Thus, the matrix should show great ability for cell adhesion, and type I collagen has been widely used for luxuriant arginine-glycine-aspartate (RGD) peptide (Sumanasinghe et al., 2009). Lohberger et al. seeded MSCs on type I collagen-coated cell culture plates, which were then subjected to continuous tension $(10 \%, 0.5 \mathrm{~Hz})$, and the tension-loaded groups show improved expression of osteogenic genes, higher calcium deposition, and more ALP when compared with the unstimulated groups (Lohberger et al., 2014). (Zhao et al., 2010) compared the effects of $0.3 \mathrm{~Hz}$ tension with different strains $(9,12$, and $15 \%)$ and found that the $12 \%$ tension group induces robust osteogenic response (Zhao et al., 2021). To explore the effects of tension in a 3D microenvironment, a novel uniaxial tension bioreactor was designed to exert tensile forces $(10 \%, 0.5 \mathrm{~Hz}$ for 7 days with $4 \mathrm{~h}$ each day) to fibrin hydrogels seeded with MSCs (Carroll et al., 2017). Results show that tension could promote the intramembranous ossification and impede the adipogenic differentiation of MSCs (Carroll et al., 2017). Therefore, the osteoinductive window of tension is mainly determined by tension strain, and 5-15\% magnitude could stimulate MSC osteogenesis.

\subsubsection{Fluid Flow Shear Stress (Perfusion and Rotation)}

FFSS is another external mechanical stimulus generated by the load of compression or tension. Load-induced FFSS could change cell shape. One classic 2D model to explore the effect of loadinduced FFSS is parallel-plate flow chamber (Yourek et al., 2010; Dash et al., 2020). Using this model, it was confirmed that shortterm continuous FFSS $\left(9\right.$ dynes $/ \mathrm{cm}^{2}$ ) for $24 \mathrm{~h}$ could promote the osteogenic differentiation of MSCs without chemically osteoinductive molecules (Yourek et al., 2010). However, for long-term intermittent FFSS, low-magnitude FFSS (such as $10 \mathrm{mPa}$, namely, 0.1 dynes $/ \mathrm{cm}^{2}$ ) is sufficient to induce osteogenesis (Dash et al., 2020). However, these strategies fail to emulate the natural ECM microenvironment; thus, various bioreactors, including rotation and perfusion bioreactors, have been developed to generate FFSS. Perfusion bioreactors have been widely used to explore the osteoinductive effects of FFSS (Filipowska et al., 2016). In the absence of biochemical cues (such as dexamethasone), FFSS $(1 \mathrm{ml} / \mathrm{min}$ ) provided by perfusion bioreactors for 16 days could dramatically promote the mineralization of MSCs within decellularized matrix/Ti meshes (Datta et al., 2006). Bjerre et al. (2008) used a perfusion bioreactor to dynamically culture MSCs seeded in silicate-substituted tricalcium phosphate scaffolds and found that FFSS $(0.1 \mathrm{ml} /$ min) for 21 days could promote the proliferation and osteogenic differentiation of MSCs. Filipowska et al. established an intermittent model $(2.5 \mathrm{ml} / \mathrm{min}$ for three times with $2 \mathrm{~h}$ per section) by using perfusion bioreactors to stimulate MSCs seeded in gelatin-coated polyurethane scaffolds and found that intermittent protocols can induce MSC osteogenesis (Filipowska et al., 2016). During dynamic perfusion culture, the expression of type $\mathrm{X}$ collagen is upregulated, suggesting that endochondral and intramembranous ossification participates in osteogenesis (Moser et al., 2018). Therefore, the osteogenic window of load-induced FFSS is mainly determined by interchangeable pressure or flow rate, and load-induced FFSS with pressure $>0.2$ dynes $/ \mathrm{cm}^{2}$ shows osteoinductive properties (Yong et al., 2020).

\subsubsection{Oscillatory Fluid Flow Shear Stress (Microvibration and Nanovibration)}

Oscillatory FFSS is another FFSS generated by oscillatory displacement without strain, which is the microscale or nanoscale form of vibration (Stewart et al., 2020; Birks and Uzer, 2021). According to amplitude, vibration can be classified into microvibration $(\leq 50 \mu \mathrm{m})$ and nanovibration $(<100 \mathrm{~nm})$

Microvibration is vibration with amplitude $\leq 50 \mu \mathrm{m}$, magnitude < $1 \mathrm{~g}$, and frequency $1-100 \mathrm{~Hz}$ (Wu et al., 2020). Frequency may determine the osteoinductive window of microvibration. Cashion et al. revealed that microvibration with a low frequency $(1 \mathrm{~Hz})$ induces chondrogenesis, whereas relatively high frequency $(100 \mathrm{~Hz})$ promotes osteogenesis (Cashion et al., 2014). Thus, low-magnitude high-frequency vibration (LMHFV) (magnitude $<1 \mathrm{~g}$, frequency $20-90 \mathrm{~Hz}$ ) is 
generally used for osteogenesis (Steppe et al., 2020). $50 \mathrm{~Hz}$ LMHFVs with different magnitudes $(0.1,0.3,0.6$, and $0.9 \mathrm{~g}$ ) were used to stimulate PDLCs, and it was found that all groups promote osteogenic differentiation, but LMHFV with $0.3 \mathrm{~g}$ peaks the osteoinduction (Zhang et al., 2015). Some studies revealed that LMHFV stimulates MSC osteogenesis in a frequency-dependent response. One study revealed that horizontal vibration at $100 \mathrm{~Hz}$ causes higher osteoinduction than that at $30 \mathrm{~Hz}$ (Pongkitwitoon et al., 2016). A previous study observed that $800 \mathrm{~Hz}$ microvibration promotes higher biomineralization and osteogenic marker expression than 0 , 30 , and $400 \mathrm{~Hz}$, but long-term stimulation of microvibration (30 min/day, 14 days) with frequencies of 30 and $400 \mathrm{~Hz}$ inhibits osteogenesis (Chen et al., 2015). Thus, stimulation duration and loading time also influence the osteogenic differentiation of MSCs. One study revealed that microvibration $(60 \mathrm{~Hz}, 1 \mathrm{~h} / \mathrm{d}$ for 5 days $)$ inhibits the mineralization and osteogenic differentiation of MSCs (Lau et al., 2011), whereas another study showed that microvibration $(30 \mathrm{~Hz}, 45 \mathrm{~min} /$ day for 21 or 40 days) could promote MSC osteogenesis (Prè et al., 2013). These results suggest that the osteoinductive window of microvibration can be determined by frequency, duration, and single loading time. For $30 \mathrm{~Hz}$ vibration, long-term duration may be effective to osteogenic differentiation. For vibration with frequencies over $60 \mathrm{~Hz}$, single loading time should be limited with $30 \mathrm{~min}$, and short-term duration ( $<7$ days) may be superior for osteogenesis.

Nanovibration refers to vibration with nanoscale amplitude $(<100 \mathrm{~nm})$. It was confirmed that nanovibration (frequency $1,000 \mathrm{~Hz}$, amplitude $10-14 \mathrm{~nm}$ ) could promote the osteogenic differentiation of MSCs in 2D condition (Nikukar et al., 2013). Another study showed that nanovibration (frequency $1,000 \mathrm{~Hz}$, amplitude $10-14 \mathrm{~nm}$ ) could stimulate the osteogenic differentiation of MSCs seeded in 3D collagen hydrogels (Tsimbouri et al., 2017). Thus, the osteoinductive window of nanovibration is a frequency of approximately $1,000 \mathrm{~Hz}$ and amplitude of $10-20 \mathrm{~nm}$.

\subsubsection{Acoustic Stimuli}

Acoustic stimuli, according to frequency, can be categorized into infrasound $(<20 \mathrm{~Hz})$, audible sound $(20-20,000 \mathrm{~Hz})$, and ultrasound $(>20,000 \mathrm{~Hz})$. Therapeutic acoustic stimuli are generally termed as ultrasound with frequency varying from 0.7 to $3.3 \mathrm{MHz}$ and low or high intensity (Zhang et al., 2017). When loaded to tissue, ultrasound could convert energy to heat via thermal effect, which may cause irreversible damage. Some nonthermal effects induced by ultrasound, including cavitation, acoustic microstreaming, acoustic radiation force, the spread of surface waves, and oscillatory FFSS may modulate the commitment of MSCs. (Esfandiari et al., 2014; Padilla et al., 2014).

Low-intensity pulsed ultrasound (LIPUS) (intensity $30-100 \mathrm{~mW} / \mathrm{cm}^{2}$, frequency $1.5 \mathrm{MHz}$, and duty cycle $20 \%$ or $100 \%)$ is a preferential strategy to reduce the thermal effect for regenerative medicine. This strategy has been approved by the United States Food and Drug Administration for the treatment of fresh fractures and established non-union (de Lucas et al., 2020).
Depending on duty cycle, continuous LIPUS (100\%) can also be used. However, one research revealed that LIPUS with $20 \%$ duty cycle shows higher osteoinductive properties toward ADSCs than LIPUS with 50\% duty cycle (Yue et al., 2013). Thus, LIPUS may be more effective than cLIPUS. Using PDLCs, the osteoinduction of LIPUS was explored, and it was found that LIPUS could stimulate osteogenic differentiation and upregulate osteocalcin, Runx2, and integrin 1, and that LIPUS with an intensity of $90 \mathrm{~mW} / \mathrm{cm}^{2}$ is more effective for osteoinduction than LIPUS with an intensity of 30 or $60 \mathrm{~mW} / \mathrm{cm}^{2}$ (Hu et al., 2014). Zhou et al. further improved the intensity of LIPUS and found that LIPUS with an intensity of $150 \mathrm{~mW} / \mathrm{cm}^{2}$ is more effective than LIPUS with intensities of $20,50,75$, and $300 \mathrm{~mW} / \mathrm{cm}^{2}$ (Zhou X. et al., 2016). These results suggest that the osteoinductive window of LIPUS is primarily determined by duty cycle and intensity, and $20 \%$ duty cycle and $90-150 \mathrm{~mW} / \mathrm{cm}^{2}$ intensity may be optimal for the osteogenic differentiation of MSCs.

Pulsed focused ultrasound (intensity $133 \mathrm{~W} / \mathrm{cm}^{2}$, frequency $1 \mathrm{MHz}$, and duty cycle 5\%) is another acoustic stimulus model with relatively minimizing thermal effect that is characterized by short term and high intensity (de Lucas et al., 2020). It could induce MSC homing (Burks et al., 2018), but whether or not it induces the osteogenic differentiation of MSCs remains unclear.

\subsection{Electromagnetic Stimuli}

Electromagnetic stimuli are also important biophysical cues that could exert effects on the fate of MSCs. Depending on physical properties, electromagnetic stimuli can be further classified into magnetic stimuli in the form of magnetic field and electric stimuli in the form of electric field and electric current.

\subsubsection{Electric Current}

Natural bone physiologically generates electric stimuli on account of non-centrosymmetric collagen after mechanical stress, which supports bone development and repair (Khare et al., 2020). Thus, external electric stimuli can be applied to MSCs for regenerative medicine. Alternating electric current is an effective external electric stimulus that induces the osteogenic differentiation of MSCs (Creecy et al., 2013). In one study, MSCs were seeded on an indium-tin-oxide-coated glass and then subjected to alternating electric current $(5-40 \mu \mathrm{A}, 5-10 \mathrm{~Hz}, 1-24 \mathrm{~h} /$ day) for 21 days without exogenous biochemical osteogenic molecules, and results showed that all setups of alternating electric current could stimulate osteogenic differentiation and inhibit chondrogenic and adipogenic differentiation (Wechsler et al., 2016). In addition, alternating electric current $(10 \mu \mathrm{A}, 10 \mathrm{~Hz}, 6 \mathrm{~h} /$ day) could reach optimized osteogenic effects (Wechsler et al., 2016). Direct current is another form of electric current, but its osteogenic effects without electric field remain unknown, which needs further research.

\subsubsection{Electric Field}

Electric field is another effective external electric stimulus to promote MSCs toward osteogenic differentiation. According to the generation pattern, electric field can be categorized into direct current electric field, capacitively coupled electric field, and inductively coupled electric field (Thrivikraman et al., 2018). 
When MSCs are exposed to electrical stimuli, the membrane potential could be altered, and hyperpolarization stimulates osteogenesis (Murillo et al., 2017; Bhavsar et al., 2019). In addition, the configuration of plasma receptors could be modulated for osteogenic differentiation (Murillo et al., 2017). Furthermore, cytoskeletal elongation and nuclear orientation could be changed by electric field for osteogenesis (Khaw et al., 2021).

Among various parameters, electric field intensity may determine the osteoinductive windows. Using osteogenic differentiation medium, a previous study applied an electric field $(2 \mathrm{mV} / \mathrm{mm}, 60 \mathrm{kHz}, 40 \mathrm{~min} /$ day $)$ to induce MSCs and found that the electric field could induce a delayed osteogenic differentiation of MSCs (Esfandiari et al., 2014). However, Hronik-Tupaj et al. reported that a $2 \mathrm{mV} / \mathrm{mm}, 60 \mathrm{kHz}$ electric field promotes chondrogenesis (Hronik-Tupaj et al., 2011). The discrepancy may be interpreted as the utilization of osteogenic molecules, which could synthetically direct electric field to promote osteogenesis. One research revealed that electric field $(0.36 \mathrm{mV} / \mathrm{mm}, 10 \mathrm{~Hz})$ alone fails to induce osteogenic differentiation but dramatically promotes MSC osteogenesis when osteogenic sulfated hyaluronan derivative is added (Hess et al., 2012). Therefore, electric field with low intensity may not promote MSCs toward osteoblasts but could synthetically improve the osteoinductive properties of biochemical molecules.

Different from low-intensity electric field, high-intensity electric field could directly promote the osteogenic differentiation of MSCs. For instance, when MSCs are subjected to electric field $(100 \mathrm{mV} / \mathrm{mm}, 1 \mathrm{~h} /$ day $)$, osteogenic differentiation occurs, and osteoinductive effects could be maintained even when the electric field is removed (Hess et al., 2012; Eischen-Loges et al., 2018). Khaw et al. utilized two electric fields (100 and $200 \mathrm{mV} / \mathrm{mm}$ ) to MSCs without biochemical osteogenic supplements, and found that both electric fields could promote the osteogenic differentiation of MSCs, and the $200 \mathrm{mV} / \mathrm{mm}$ electric field was optimized (Khaw et al., 2021). Ravikumar et al. designed an electric field device where a static potential $(15 \mathrm{~V})$ was loaded to parallel electrodes with a space of $15 \mathrm{~mm}$ (Ravikumar et al., 2017). MSCs were seeded to $\mathrm{HA}-\mathrm{CaTiO} 3$ composites and then exposed to electric field for $10 \mathrm{~min} /$ day without osteogenic molecules, and results showed that the electric field could dramatically improve the osteogenic markers (Ravikumar et al., 2017). These studies suggest that an electric field with an intensity over $100 \mathrm{mV} /$ $\mathrm{mm}$ may be needed for MSC osteogenesis without biochemical osteogenic molecules.

\subsubsection{Magnetic Field}

Exposure of MSCs to magnetic field may directly deform their plasma membrane, which causes cytoskeleton remodeling, improves cell viability, and promotes differentiation (Santos et al., 2015). Magnetic biomaterials should be introduced to culture systems, including magnetic particles and substrates, to enhance the effects of magnetic field. Boda et al. fabricated a series of hydroxyapatite- $\mathrm{Fe}_{3} \mathrm{O}_{4}$ magnetic substrates with different magnetization and then applied a periodic magnetic field $(100 \mathrm{mT})$ to these magnetic substrates seeded with MSCs, and they found that all magnetic substrates combined with magnetic field could promote the osteogenic differentiation of MSCs (Boda et al., 2015). Magnetic particles can be also used to transform magnetic stimuli into mechanical stimuli. Magnetic particles coupled with magnetic field (14.7 or $21.6 \mathrm{mT}$ ) were used to stimulate ADSCs, and it was found that low-density magnetic field $(14.7 \mathrm{mT})$ with intermittent short-term exposure (2 days) favors adipogenic differentiation, whereas high-density magnetic field $(21.6 \mathrm{mT})$ promotes osteogenesis in all exposure profiles including continuous or intermittent long-term exposure (7 days) and intermittent short-term exposure (2 days) (Labusca et al., 2020). To further control the effects of magnetic particles, magnetic particles are generally functionalized by antibodies or peptides, which could directly exert pico-newton level forces to mechanosensitive plasma membrane receptors under magnetic field, which could then induce the osteogenic differentiation of MSCs (Kanczler et al., 2010; Hu et al., 2013). For example, Henstock et al. used either the antibody of transmembrane ion channel stretch-activated potassium channel (TREK-1) or RGD peptide to functionalize magnetic nanoparticles (Henstock et al., 2014). Then, an oscillating magnetic field $(25 \mathrm{mT})$ was loaded to collagen hydrogels containing MSCs and functionalized magnetic nanoparticles to generate a force of $4 \mathrm{pN}$, and results showed that functionalized magnetic nanoparticles could promote matrix mineralization (Henstock et al., 2014). The osteogenic window of magnetic field may be determined by magnetic flux density (21.6-100 mT), and the quantity and functionalization of magnetic nanoparticles affect their osteogenic effectiveness.

\subsubsection{Electromagnetic Field}

Electromagnetic field is a combination of electric and magnetic fields, and pulsed electromagnetic field (PEMF) has been clinically used to delay osteoporosis and bone fracture repair (Sun et al., 2009). When loaded to MSCs, PEMF without osteogenic molecules inherently induces osteogenic differentiation and impedes angiogenic differentiation, and the osteogenic effects can be further enhanced by additional osteogenic molecules (Ongaro et al., 2014; Lu et al., 2015). For example, Arjmand et al. seeded ADSCs to PCL nanofibrous scaffolds, which were then exposed to PEMF $(1 \mathrm{mT}, 50 \mathrm{~Hz})$ with or without osteogenic medium (Arjmand et al., 2018). Results revealed that the osteogenic effects of PCL scaffolds with PEMF are comparable with those of PCL scaffolds with osteogenic medium and that PCL scaffolds with PEMF and osteogenic medium show enhanced osteogenic differentiation (Arjmand et al., 2018). PEMF has been also verified to stimulate MSC proliferation (Tsai et al., 2009; Sun et al., 2010). Therefore, PEMF shows great promise for bone tissue engineering.

Among various parameters describing PEMF, magnetic flux density and frequency may determine the osteoinductive window of PEMF. For magnetic flux density, Jazayeri et al. compared two PEMFs ( 0.1 and $0.2 \mathrm{mT}$ ) and found that the expression of osteogenic markers, such as RUNX2 and OCN, is higher under $0.2 \mathrm{mT}$ PEGF than under 0.1 mT PEGF (Jazayeri et al., 2017). Esposito et al. used a device that could generate $1.8-3 \mathrm{mT}$ PEGF to induce MSCs and observed osteogenic differentiation (Esposito et al., 2012). Therefore, magnetic flux density is generally limited to $0.1-3 \mathrm{mT}$, but the optimal density remains 


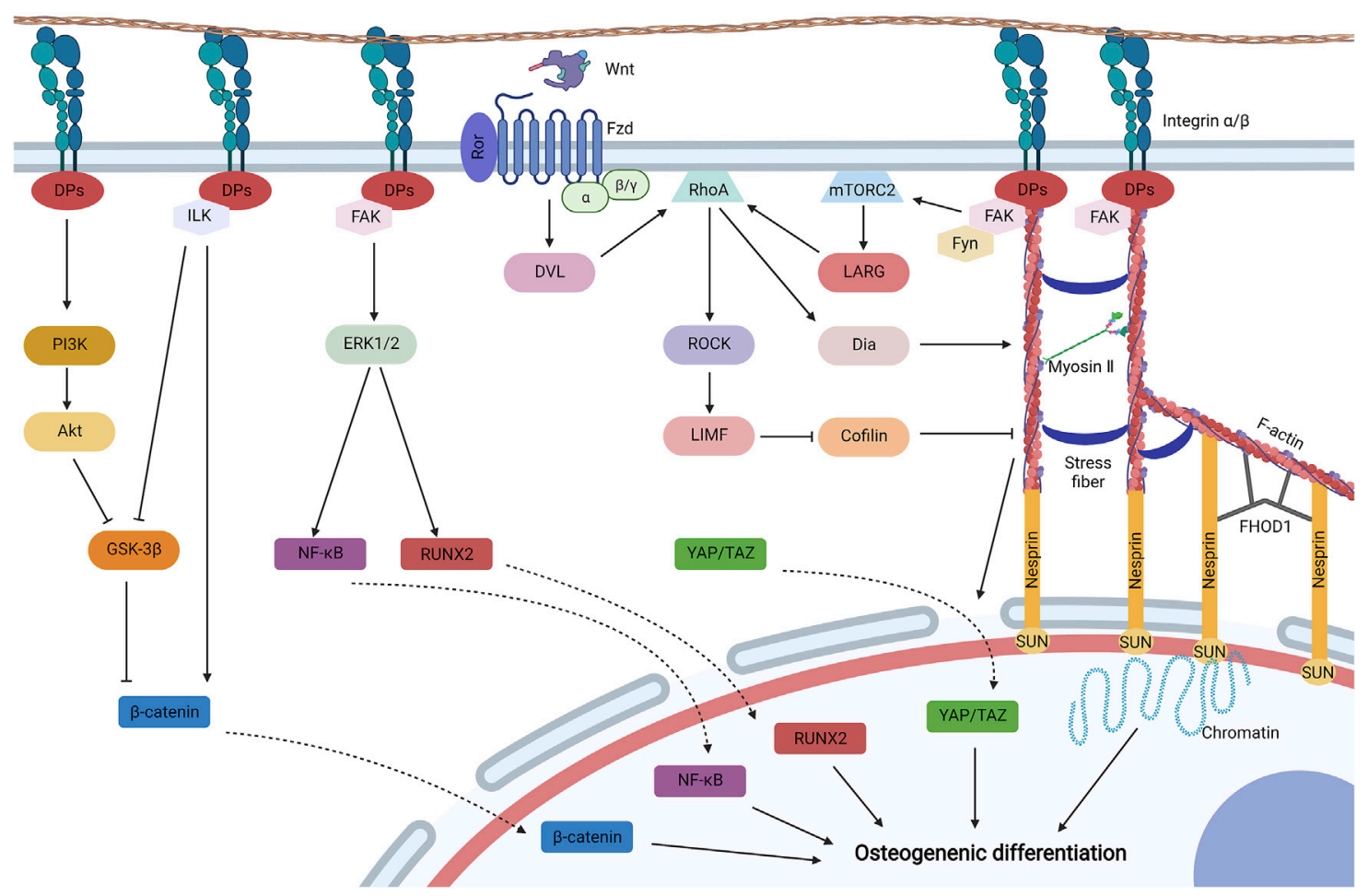

FIGURE 3 | Self-biophysical transduction for mesenchymal stem cell (MSCs) osteogenesis, including biophysical sensitive ion channels, primary cilium, and biophysical sensitive. When MSCs are exposed to biophysical stimuli, various biophysical sensitive ion channels on the plasma membrane may be activated for the influx of $\mathrm{Ca}^{2+}$, which include voltage-gated calcium channels (VGCC), stretch-activated calcium channels (SACCs), Piezo, transient receptor potential vallanoid 1 (TPRV1), and TPRV4. TPRV4 is located at high-strain regions, especially primary cilium. Biophysical stimuli can also stimulate the release of $\mathrm{Ca}^{2+}$ from the endoplasmic reticulum to the cytoplasm by uncanonical Wnt-Ca2+ signaling. In specific, Wnt ligand binds to the Frizzled (Fzd)/receptor tyrosine kinase-like orphan receptor (Ror) complex to activate Dishevelled (Dvl), which then activates phospholipase C (PLC) to produce inositol 1,4,5-trisphosphate (IP3). IP3 could activate IP3 receptor for the release of $\mathrm{Ca}^{2+}$ from the endoplasmic reticulum. The increased $\mathrm{Ca}^{2+}$ in the cytoplasm may promote osteogenic differentiation by activating calcineurin/Nuclear Factor of Activated Cells (NF-AT) signaling and initiating extracellular signal-related kinase 1/2 (ERK1/2) by focal adhesion kinase (FAK) to regulate the activity of $\beta$-catenin. Biophysical stimuli could regulate the primary cilium containing intraflagellar transport protein 88 (IFT88) for MSC osteogenesis by regulating length or Gpr161/adenylyl cyclase 6 (AC6) signaling, and the activation of AC6 promotes the synthesis of cyclic adenosine $3^{\prime}, 5^{\prime}$-monophosphate (cAMP), which then promotes the osteogenic differentiation of MSCs by Hedgehog $(\mathrm{Hb})$ signaling and proteinkinase A (PKA) signaling. Biophysical stimuli could also promote some long noncoding RNAs (IncRNAs) to inhibit some microRNAs (miRNAs) for osteogenic differentiation. Created with BioRender.com.

unknown. On the other hand, for frequency, MSCs were exposed to PEMFs with different frequencies $(5,25,50,75,100$, and $150 \mathrm{~Hz}$ ), and all groups showed osteogenic differentiation, but with the increase of frequencies, osteogenic differentiation initially enhanced and then peaked at $50 \mathrm{~Hz}$, which decreased until $150 \mathrm{~Hz}$ (Luo et al., 2012). Lim et al. also found that PEMF with $50 \mathrm{~Hz}$ shows improved osteogenic differentiation compared with those with 10 and $100 \mathrm{~Hz}$ (Lim et al., 2013). Therefore, the frequency of PEMF should be limited to $10-150 \mathrm{~Hz}$, and $50 \mathrm{~Hz}$ is preferable.

\section{OSTEOINDUCTIVE MECHANISMS OF BIOPHYSICAL STIMULI FOR BONE TISSUE ENGINEERING}

When distinctive biophysical stimuli are subjected to MSCs, they will be ultimately loaded by or transferred to either mechanical or electromagnetic stimuli. Thus, mechanotransduction and electrocoupling have been separately proposed to interpret their molecular mechanisms. Considering that both signaling show high comparability and that they simultaneously occur in physiological microenvironment, we propose a new concept of biophysical transduction that integrates mechanotransduction and electrocoupling. Biophysical transduction mainly includes three stages: sensing, transmission, and regulation. According to the sensing pattern of MSCs, biophysical transduction can be further categorized into self-biophysical transduction, cell-matrix biophysical transduction, and cell-cell biophysical transduction.

\subsection{Self-Biophysical Transduction}

Self-biophysical transduction refers to biophysical sensing coupled with transmission and regulation by structures that do not adhere with biomaterials and adjacent cells, which mainly include biophysical-sensitive ion channels and primary cilium. And some biophysical-sensitive ribose nucleic acids (RNAs) are also upregulated by biophysical stimuli to regulate the osteogenic differentiation of MSCs (Figure 3). 


\subsubsection{Biophysical-Sensitive Ion Channels}

A plethora of biophysical-sensitive ion channels exist on the surface of plasma membrane or endoplasmic reticulum, which can be activated by distinctive biophysical stimuli for $\mathrm{Ca}^{2+}$ influx to induce the osteogenic differentiation of MSCs. When a sufficiently giant electric field is applied to plasma membrane and generate a large transmembrane potential difference (over $100 \mathrm{mV}$ ), voltage-gated calcium channels can be directly activated because of plasma depolarization (Thrivikraman et al., 2018). Electric field and mechanical tension could directly promote the influx of $\mathrm{Ca}^{2+}$ by activating stretchactivated calcium channels (Cho et al., 1999; Kearney et al., 2010). In addition, biophysical-sensitive Piezol for $\mathrm{Ca}^{2+}$ influx could be initiated by mechanical stimuli, such as HP (Sugimoto et al., 2017). Transient receptor potential vallanoid 1 (TRPV1) can be activated by nanovibration (Tsimbouri et al., 2017), whereas TRPV4 can be activated by load-induced FFSS, which is mainly located at high-strain regions (especially primary cilium) (Corrigan et al., 2018; Eischen-Loges et al., 2018). The initiation of these biophysical-sensitive ion channels increases $\mathrm{Ca}^{2+}$ concentration in the cytoplasm, which may initiate calmodulin/calcineurin signaling (Kapat et al., 2020). Calcineurin frees the phosphate group from phosphorylated nuclear factor of activated cells (NF-AT), and dephosphorylated NF-AT then shuttles to the nucleus and interacts with other transcription factors to induce the osteogenic differentiation of MSCs (Khare et al., 2020). In addition, increased $\mathrm{Ca}^{2+}$ in the cytoplasm initiates protein kinase $C$ and extracellular signal-related kinase $1 / 2$ (ERK1/2), which then regulate the activity of $\beta$-catenin to promote MSC osteogenesis (Tsimbouri et al., 2017).

The increase in $\mathrm{Ca}^{2+}$ concentration in the cytoplasm can also be mediated by $\mathrm{Ca}^{2+}$ release from the endoplasmic reticulum, which may be related to noncanonical Wnt- $\mathrm{Ca}^{2+}$ signaling (Bertrand et al., 2020). Biophysical stimuli promote the expression of Wnt (Chen et al., 2019; Fu et al., 2020). Thus, secreted Wnt may interact with a transmembrane receptor Frizzled (Fzd) coupled with receptor tyrosine kinase-like orphan receptor (Ror) to promote the activity of phospholipase C (PLC) by activated Dishevelled (Dvl), and then PLC degrades phosphatidylinositol 4,5-bisphosphate in the cell membrane to obtain inositol 1,4,5-trisphosphate (IP3), which moves to the endoplasmic reticulum and binds to IP3 receptors to promote $\mathrm{Ca}^{2+}$ release (Thrivikraman et al., 2018). Moreover, $\mathrm{Ca}^{2+}$ channels on the endoplasmic reticulum may be directly modulated by electromagnetic stimuli because of the change in configuration, which also promotes the increase in $\mathrm{Ca}^{2+}$ concentration in the cytoplasm (Khare et al., 2020). Therefore, endoplasmic reticulum-derived $\mathrm{Ca}^{2+}$ may participate in the osteoinduction of biophysical stimuli.

\subsubsection{Primary Cilium}

Primary cilium is a biophysical-sensitive organelle based on immotile microtubule and appears from the cytomembrane surface (Delaine-Smith and Reilly, 2012). Primary cilium could sense mechanical and electromagnetic stimuli to induce the osteogenic differentiation of MSCs (Hoey et al., 2012; Chen et al., 2016). The osteoinductive mechanism of primary cilium may be related to its length regulation and ciliary receptors or molecules. One research revealed that topography influences the length of primary, and reduced length promotes the nuclear translocation of $\beta$-catenin (McMurray et al., 2013). Thus, specific biophysical stimuli may lower the length of primary cilium for MSC osteogenesis. Gpr161 is a biophysical-sensitive G protein-coupled receptor (GCPR) localized to primary cilium containing intraflagellar transport protein 88 (IFT88), which is essential for cilium formation (Johnson et al., 2021). After being subjected to biophysical stimuli, the GCPR may activate ciliary localized adenylyl cyclase 6 (AC6) for the synthesis of cyclic adenosine $3^{\prime}, 5^{\prime}$-monophosphate (cAMP), which then activates Hedgehog signaling for MSC osteogenesis (Johnson et al., 2018; Johnson et al., 2021). cAMP may also initiate protein kinase A to induce the osteogenic differentiation of MSCs (Johnson et al., 2018). Further studies should focus on other receptors or molecules localized to primary cilium from MSCs and their biophysical transduction and osteoinductive mechanisms.

\subsubsection{Biophysical-Sensitive RNAs}

After being subjected to biophysical stimuli, MSCs could express some RNAs, including microRNAs (miRNAs) and long noncoding RNAs (lncRNAs), to regulate osteogenic differentiation. One research revealed that tension promotes the expression of lncRNA-MEG3, which then inhibits the expression of miRNA-140-5p for MSC osteogenesis (Zhu et al., 2021). Another research revealed that lncRNA H19 is upregulated to impede miRNA-138, and decreased miRNA-138 may recover the expression of focal adhesion kinase (FAK), which participates in cell-matrix biophysical transduction ( $\mathrm{Wu}$ et al., 2018). miRNA-132-3p and miR-129-5p are also inhibited when MSCs are subjected to biophysical stimuli for osteogenesis ( $\mathrm{Hu}$ et al., 2020; Wu et al., 2021). Although biophysical-sensitive RNAs exert critical effects in biophysical regulation, related studies are limited, which need further attention.

\subsection{Cell-Matrix Biophysical Transduction}

Cell-matrix biophysical transduction refers to biophysical sensing coupled with transmission and regulation by structures that adhere to biomaterials. Cell adhesion is mainly induced and regulated by integrin and focal adhesion (Figure 4).

\subsubsection{Integrin Signaling}

Integrin is a transmembrane heterodimer that serves as a receptor to bind with specific ligands from external microenvironment (Thompson et al., 2012). When MSCs adhere to biomaterials and subjected to biophysical stimuli, multiple integrin signaling can be activated for the osteogenic differentiation of MSCs. FAK, under biophysical stimuli, may be recruited to integrin and undergo autophosphorylation (Bertrand et al., 2020), which then activates mitogen-activated protein kinases (MAPKs), such as ERK1/2 and P38 (Liu et al., 2014; Niu et al., 2017; Chang et al., 2019). Activated or phosphorylated MAPKs then induce the phosphorylation of transcription factors, such as RUNX2, to promote the osteodifferentiation of MSCs (Chang et al., 2019). Activated ERK1/2 also phosphorylates nuclear factor 


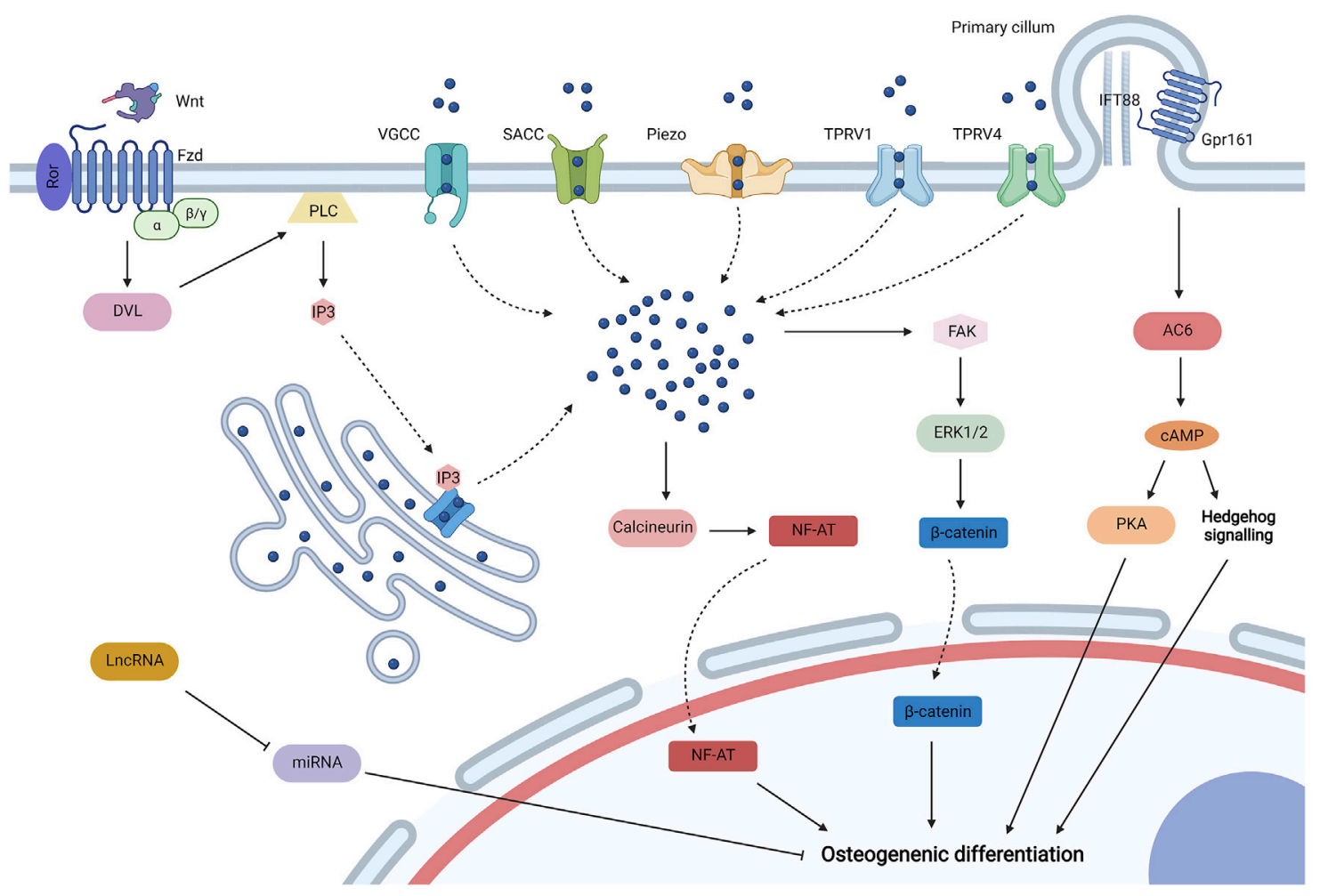

FIGURE 4 | Cell-matrix biophysical transduction for MSC osteogenesis, including integrin signaling and focal adhesion. Integrins generally induce cell adhesion, and integrin signaling may be activated for the osteogenic differentiation of MSCs under biophysical stimuli. Integrin activates phosphatidylinositol 3-kinase (PI3K) to

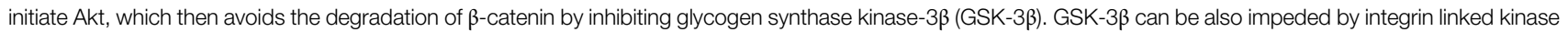
(ILK) initiated by integrin. Activated ILK can also promote the cytoplastic accumulation of $\beta$-catenin by dissociating $\beta$-catenin from cadherin. $\beta$-catenin is then translocated to the nucleus for osteogenic differentiation. Integrin can also initiate FAK to activate mitogen-activated protein kinase (MAPK) (such as ERK1/2) to phosphorylate transcription factors, such as Runt-related transcription factor 2 (Runx2) and nuclear factor $\mathrm{kB}$ (NF-kB), which then shuttles to the nucleus for MSC osteogenesis. Biophysical stimuli can be directly transduced by the structure of focal adhesion-cytoskeleton-nucleus to promote chromatin for gene expression, which is composed of integrin, docking proteins (DPs), recruited FAK, F-actin, myosin II, stress fiber, the Linker of Nucleoskeleton and Cytoskeleton (LINC) complex (nesprin and SUN protein), and formin homology 1/formin homology 2 domain containing protein 1 (FHOD1). The structure is mainly regulated by ras homolog family member $\mathrm{A}$ (RhoA) signaling. RhoA can be activated by leukemia-associated Rho guanine nucleotide exchange factor (LARG), which is initiated by the recruitment of Fyn and FAK to initiate mammalian target of rapamycin complex 2 (mTORC2). RhoA can be also activated by initiated Dvl by the binding of Wnt to the Ror/Fzd complex. Activated RhoA initiates LIM kinase (LIMK) by stimulating Rho-associated protein kinase (ROCK), which could inhibit cofilin to avoid F-actin severing. Activated RhoA also promotes the formation and contraction of the structure by initiating Diaphanous (Dia). Furthermore, contracted cytoskeleton may diminish the mechanical resistance for the translocation of Yes-associated protein (YAP) and transcriptional co-activator with PDZ-binding motif (TAZ) to the nucleus for osteogenesis. Created with BioRender.com.

$\kappa \mathrm{B}(\mathrm{NF \kappa} \mathrm{B})$, which upregulates integrin $\beta 1$ in a feedback model and promotes the expression of BMP-2 for osteogenesis (Liu et al., 2011; Liu et al., 2014). Another research revealed that activated ERK1/2 inhibits adipogenesis by downregulating the expression of BMP-4 (Lee et al., 2012). Moreover, biophysical stimuli may activate integrin-linked kinase (ILK) by integrin, which inhibits $\mathrm{N}$-cadherin to release $\beta$-catenin and glycogen synthase kinase-3 $\beta$ (GSK-3 $\beta$ ) to avoid the degradation of $\beta$-catenin. Then, $\beta$-catenin shuttles to the nucleus to induce the osteogenic differentiation of MSCs (Niu et al., 2017). Furthermore, integrin could be stimulated by biophysical stimuli to activate Akt by phosphatidylinositol 3-kinase (PI3K), which then activates GSK-3 $\beta$ to inhibit the degradation of $\beta$-catenin for MSC osteogenesis (Song et al., 2017; Sun et al., 2018). Osteoinductive mechanisms of integrin signaling by biophysical stimuli can be mainly interpreted as
FAK/MAPK signaling, ILK/ $\beta$-catenin signaling, and PI3K/Akt/ $\beta$-catenin signaling. In addition, crosslinking exists among distinctive integrin signaling.

\subsubsection{Focal Adhesion-Cytoskeleton-Nucleus}

In addition to indirect integrin signaling to induce osteogenesis, integrins also participate in the formation of focal adhesion to transfer biophysical stimuli directly to contractible cytoskeleton and nucleus, which is generally known as bundles of clustered integrins (Dalby et al., 2014). At the site of focal adhesion, the cytoplasmic tail of integrin is linked to the (F)-actin cytoskeleton by talin and vinculin, which is stabilized by other docking proteins, including zyxin, actinin, and p130Cas (Bertrand et al., 2020). Adjacent actins generate prestress by the bundling of stress fibers (such as a-actinin) and myosin II (Wang et al., 2009). The tail of actin is linked to the nuclear 
envelope by the Linker of Nucleoskeleton and Cytoskeleton (LINC) complex, which is composed of nesprin (nesprin1 or nesprin2) and SUN (Bouzid et al., 2019). Moreover, formin homology $1 /$ formin homology 2 domain containing protein 1 binds to multiple sites among nesprin and actin to enhance the association (Birks and Uzer, 2021). Furthermore, SUN proteins bind to lamins to form the lamina, and lamina coupled with G-actin and myosin may assemble into the nucleoskeleton, which connects to chromatin and deoxyribonucleic acid (DNA) (Wang et al., 2009). Therefore, the structure of focal adhesioncytoskeleton-nucleus allows biophysical stimuli to be transferred from outside to DNA and directly activates gene expression (Wang et al., 2009). When MSCs are subjected to biophysical stimuli, autophosphorylated FAK is recruited to focal adhesion and connects to integrin $\beta$ by talin and paxillin and promotes cytoskeletal contraction, which then promote MSC osteogenesis (Hao et al., 2015; Bertrand et al., 2020).

Cytoskeletal stabilization and contraction are mainly regulated by biophysical-sensitive ras homolog family member A (RhoA) signaling. And biophysical stimuli could promote the osteogenic differentiation of MSCs by RhoA signaling (Arnsdorf et al., 2009a; Zhao et al., 2015). The activation of RhoA is related to focal adhesion and uncanonical Wnt/RhoA signaling. After biophysical stimuli, kinase Fyn and FAK are recruited to focal adhesion, which synthetically initiate mammalian target of rapamycin complex 2 to activate RhoA, which may be related to the activation of leukemia-associated Rho guanine nucleotide exchange factor (Thompson et al., 2013; Thompson et al., 2018). Moreover, biophysical stimuli could stimulate MSCs to upregulate Wnt5a and Ror2 (Arnsdorf et al., 2009b; Shi et al., 2012). Wnt ligand binds to the complex of Ror and Fzd to initiate Dvl, which could also activate RhoA (Bertrand et al., 2020). The activated RhoA then initiates Rho-associated protein kinase (ROCK) and subsequently LIM kinase (LIMK), which inactivate or phosphorylate cofilin to diminish its effects of severing F-actin (Hayakawa et al., 2011; Bertrand et al., 2020). The structure of focal adhesion-cytoskeleton-nucleus can be also enhanced by the initiation of Diaphanous via RhoA (Bertrand et al., 2020).

Yes-associated protein (YAP) and transcriptional co-activator with PDZ-binding motif (TAZ) are biophysical-sensitive transcriptional activators for MSC osteogenesis. When MSCs are subjected to biophysical stimuli, YAP/TAZ are activated and then translocate to the nucleus, where they interact with various transcription factors to promote osteogenesis (Kim et al., 2014; Qian et al., 2017; Li et al., 2020). The regulation of YAP/ TAZ stimulated by biophysical stimuli may undergo a Hippo/ LATS-independent signaling pathway (Dupont et al., 2011). Recent studies have revealed that the nuclear translocation of $\mathrm{YAP} / \mathrm{TAZ}$ is related to the structure of focal adhesioncytoskeleton-nucleus (Driscoll et al., 2015). When biophysical stimuli are loaded to MSCs, forces cause focal adhesion to the nucleus by the cytoskeleton to open the size of nuclear pores relatively. Thus, the mechanical resistance to transfer molecules is lowered, allowing for YAP/TAZ translocate to the nucleus (Elosegui-Artola et al., 2017).

\subsection{Cell-Cell Biophysical Transduction}

Cell-cell biophysical transduction refers to biophysical sensing coupled with transmission and regulation by direct contact by structures from adhering with adjacent cells (cadherin and Notch receptor) (Figure 5). Bioactive factors under biophysical stimuli by the autocrine and paracrine network could also exert effects via indirect interaction (Figure 6).

\subsubsection{Adhesion Junction}

Intercellular adhesion junction is directed by calcium-dependent cadherins, and MSCs express neural (N-) cadherin and epithelial (E-) cadherin (Qin L. et al., 2020). The classical structure of cadherins can be divided into three domains: an extracellular domain to direct intercellular adhesion, a single-pass transmembrane domain, and a cytoplasmic domain to bind multiple proteins including $\beta$-catenin and $\alpha$-catenin (Leckband and de Rooij, 2014). When MSCs are subjected to mechanical stimuli (such as load-induced FFSS) or electromagnetic stimuli (such as PEMF), $\beta$-catenin disassociates from $\mathrm{N}$-cadherin or E-cadherin to the cytoplasm, respectively, which then moves to the nucleus to induce osteogenic differentiation (Arnsdorf et al., 2009b; Zhang et al., 2020).

The $\beta$-catenin signaling pathway is generally regulated by canonical Wnt signaling, which shows biophysical sensitivity. When MSCs are subjected to electromagnetic stimuli for osteogenesis, the expression levels of Wnt, low-density lipoprotein receptor-related protein (Lrp), and $\beta$-catenin are upregulated, suggesting that canonical Wnt signaling exerts critical effects in osteogenic differentiation (Chen et al., 2019; Fu et al., 2020). Secreted Wnt binds to the complex formed by Fzd and LRP 5/6, which then activates Dvl to impede the phosphorylation and degradation of $\beta$-catenin mediated by the axin/adenomatous polyposis coli/GSK-3 $\beta / \mathrm{CK} 1$ (Axin/APC/ GSK-3 $\beta / C K 1)$ complex (Schupbach et al., 2020). Then, $\beta$-catenin shuttles to the nucleus to activate osteogenic Runx2 and osterix (Benayahu et al., 2019). The activation of canonical Wnt signaling by biophysical stimuli could also inhibit MSC adipogenesis by downregulating the expression of peroxisome proliferator-activated receptor $\gamma$ (Sen et al., 2008; Case et al., 2013).

\subsubsection{Notch Signaling}

Exerting critical effects on stem cell specification and bone development, Notch signaling is an intercellular conversed pathway that is activated by a surface ligand [Delta-like (Dll)1, 3 , or 4 and Jagged (JAG) 1 or 2] from adjacent cells to bind their Notch receptor (Notch1, 2, 3, or 4) (Bagheri et al., 2018). After initiation, two types of proteases (ADAM-family metalloproteases and presenilin- $\gamma$-secretase complex) exert effects at an activated Notch receptor to release the Notch intracellular domain (NICD). The NICD then forms a complex with the DNA-binding CSL protein after translocating to the nucleus, which recruits coactivator Mastermind to transcript related genes. When MSCs are subjected to PEMF, Notch-4 receptor, Dll-4 ligand, and related genes (Hey1, Hes1, and Hes5) are upregulated, and inhibitors of Notch signaling diminish the osteoinducutive effects of PEMG 


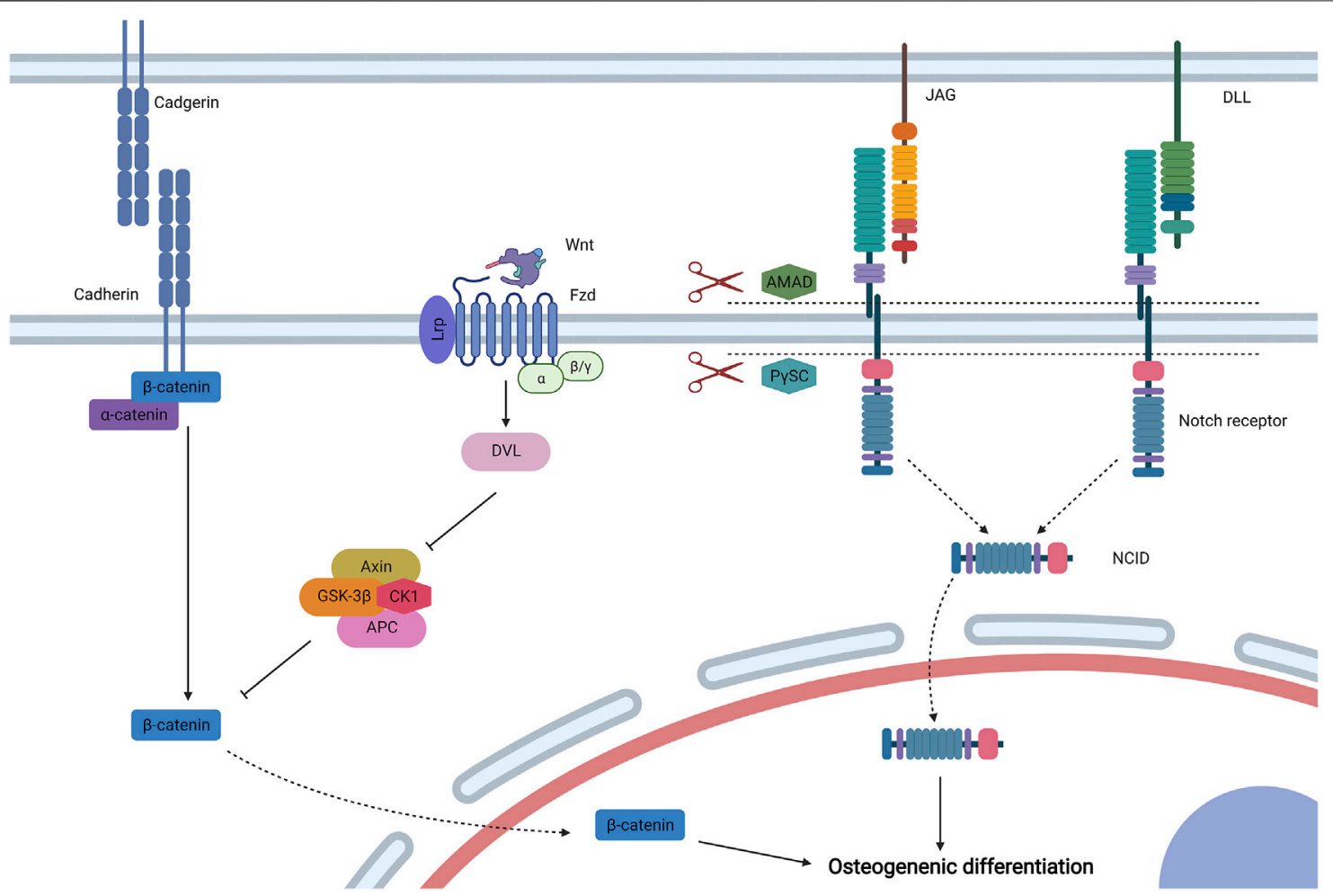

FIGURE 5 | Cell-cell biophysical transduction for MSC osteogenesis: direct interaction. Cadherin is a main molecule to induce direct intercellular interaction, and $\alpha$-catenin and $\beta$-catenin are linked to the cytoplasmic section of cadherin. Under biophysical stimuli, $\beta$-catenin dissociates from cadherin to cytoplasm. In addition, $\beta$-catenin is mainly regulated by biophysical sensitive canonical Wnt signaling. Wnt binds to the lipoprotein receptor-related protein (Lrp)/Fzd complex and activates Dvl to

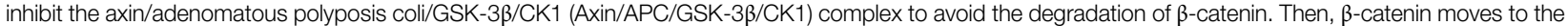
nucleus to promote MSC osteogenesis. The osteoinduction of direct cell-cell biophysical transduction can be induced by Notch signaling, which is mediated by Notch receptors and Delta-like (DII) or Jagged (JAG) ligands. Under biophysical stimuli, two proteases, including ADAM-family metalloproteases and presenilin- $\gamma$-secretase complex $(\mathrm{P} \gamma \mathrm{SC})$, release Notch intracellular domain (NICD) form Notch receptor, which then moves to the nucleus for osteogenic differentiation. Created with BioRender.com.

(Bagheri et al., 2018). Thus, Notch signaling is involved in the osteogenic differentiation of MSCs stimulated by electromagnetic signaling. Another research also reported that mechanical stimuli could initiate JAG1-mediated Notch signaling to promote MSC osteogenesis, which is controlled by the inhibition of endogenous histone deacetylase 1 (Wang et al., 2016a).

\subsubsection{Autocrine and Paracrine Network}

After being subjected to biophysical stimuli, MSCs secrete various biophysical-sensitive molecules, including BMP-2 (Liu et al., 2011; Rui et al., 2011), vascular endothelial growth factor (Lee et al., 2017), insulin-like growth factor 1 (Tahimic et al., 2016), transforming growth factor- $\beta$ (TGF- $\beta$ ) (Li et al., 2015b), and migration inhibitory factor (Yuan et al., 2016), which could biochemically promote MSC osteogenesis by autocrine. In addition, bone regeneration in vivo is an extremely complex process that involves osteogenesis, angiogenesis, osteoclastogenesis, neurogenesis, and immune regulation; thus, a paracrine network possibly stimulates osteogenesis among various cells when subjected to biophysical stimuli. Paracrine factors from stimulated osteocytes promote the osteogenic differentiation of MSCs but do not induce the osteogenic differentiation of osteoblasts (Hoey et al., 2011; Brady et al., 2015). Another study found that exosomes containing miRNA181b-5p from stimulated osteocytes enhance the osteogenic differentiation of PDLCs by BMP-2/RUNX2 (Lv et al., 2020). Stimulated osteocytes also secrete nitric oxide and relatively more osteoprotegerin than NF- $\kappa B$ ligand (RANKL) to inhibit osteoclastogenesis (Tan et al., 2007; You et al., 2008). Moreover, exosomes from stimulated MSCs impede osteoclastogenesis by weakening the activity of NF- $\kappa B$ signaling (Xiao et al., 2021). Although paracrine factors from stimulated osteoblasts fail to promote the osteogenic differentiation of MSCs, they inhibit the formation of osteoclasts (Tan et al., 2007). Furthermore, Dong et al. (2021) found that tension could activate macrophages to M2 phenotype, secrete anti-inflammatory factors (such as TGF- $\beta$ and interleukins-10), and promote the nuclear translocation of YAP to generate BMP-2 for the osteogenic differentiation of MSCs. Altogether, the autocrine and paracrine network may ultimately promote the osteogenic differentiation of MSCs in vivo, which further promote bone repair. 


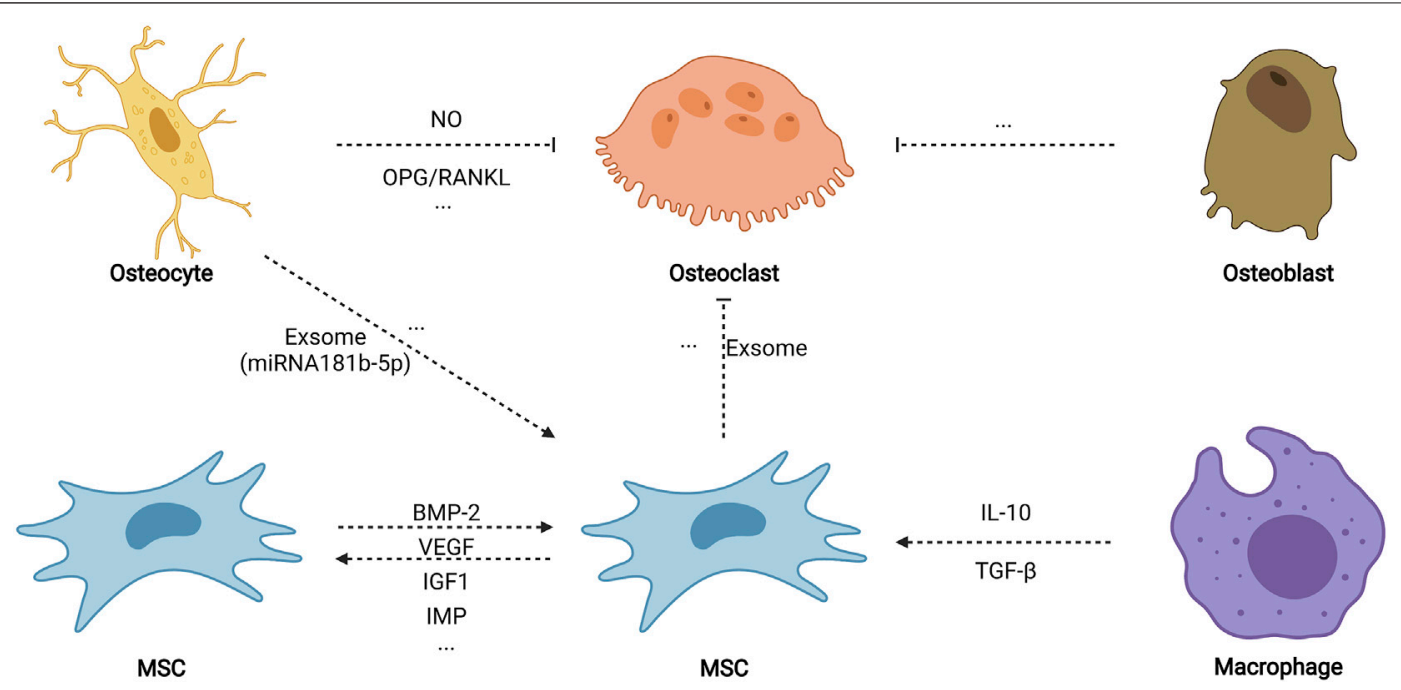

FIGURE 6 | Cell-cell biophysical transduction for MSC osteogenesis: indirect autocrine and paracrine. Indirect cell-cell biophysical transduction is mainly induced by the autocrine and paracrine network. Under biophysical stimuli, MSCs may sensitively secret some autocrine proteins to promote osteogenesis, including BMP-2, VEGF, IGF1, TGF- $\beta$, and MIF. MSCs also secrete paracrine factors (such as exosome) to inhibit osteoclastogenesis. Osteocytes release nitric oxide (NO) and relatively more osteoprotegerin (OPG) than NF-kB ligand (RANKL) to impede osteoclastogenesis. Paracrine factors from osteoblasts also diminish osteoclastogenesis. Moreover, paracrine factors (such as exosome containing miRNA181b-5p) stimulate the osteogenic differentiation of MSCs. Biophysical stimuli also stimulate macrophage (phenotype 2) to promote MSC osteogenesis. Created with BioRender.com.

\section{APPLICATIONS OF BIOPHYSICAL STIMULI FOR BONE TISSUE ENGINEERING}

For critical bone defects, bone tissue engineering is committed to replace or surpass autografts for surgical bone repair. Given their osteoinductive properties, distinctive biophysical stimuli can be used as the fourth pillar to improve traditional bone tissue engineering for bone healing. Biophysical stimuli can be integrated to bone tissue engineering by three methodologies: preconstructed scaffolds with osteoinductive properties, TEBGs, and postoperative biophysical stimuli loading strategies.

\subsection{Preconstructed Scaffolds With Osteoinductive Properties}

Biomaterial scaffolds comprise an important pillar for bone tissue engineering, but most current scaffolds are used to provide osteoconductivity without osteoinductivity. Thus, most studies focused on the introduction of bioactive molecules to scaffolds by various strategies. However, these methods fail to construct scaffolds with multi-environment for different regenerative purposes. Thus, preconstructed scaffolds by biophysical stimuli show great promise for novel bone tissue engineering because these scaffolds show inherent osteoinductivity, which also support the construction of multi-microenvironment. Preconstructed scaffolds include stiffness-improved scaffolds, topography-modified scaffolds, piezoelectric scaffolds, and magnetically actuated scaffolds.

\subsubsection{Stiffness-Improved ECM-like Scaffolds}

ECM-like scaffolds are those scaffolds that are structurally analogous to natural nanoscale natural ECM, which can be obtained by electrospinning or hydrogelation. However, the application of ECM-like scaffolds is generally limited by their unsatisfying stiffness. Thus, various strategies, including chemical and physical crosslinking, have been developed to enhance their rigidity. Chemical crosslinking improves stiffness by introducing chemical crosslinkers (such as genipin) and specific chemical groups, whereas physical crosslinking enhances rigidity by constructing an interpenetrating network or physical crosslinkers, such as inorganic particles and graphene (Zhang et al., 2016). When the stiffness of organic scaffolds is improved, these scaffolds could even stimulate the osteogenic differentiation of MSCs without osteogenic molecules. For example, elastin-like polypeptides (ELPs) are recombinant biomaterials that could assemble into organic scaffolds when the temperature reaches over inverse phase transition temperature (Ding et al., 2020). Glassman et al. (2016) utilized telechelic oxidative coupling to chemically interlink or oxidate ELPs containing cysteine, and the stiffness of ELP scaffolds is obviously enhanced, varying from $5 \mathrm{kPa}$ to over $1 \mathrm{MPa}$. When seeded on the toughened scaffolds, MSCs could be induced to undergo osteogenic differentiation without bioactive molecules. Raftery et al. introduced chitosan to collagen to construct organic scaffolds with an interpenetrating network and found that the modulus is improved and that the interpenetrating scaffold (collagen/chitosan ratio 75/25) could dramatically promote calcium deposition and sulfated glycosaminoglycan production (Raftery et al., 2016). Thus, stiffness-improved scaffolds may be superior to unoptimized organic scaffolds.

\subsubsection{Topography-Modified Scaffolds}

Modifying topography is another methodology for osteoinductive preconstructed scaffolds. The outer and inner 
surfaces can be modified for different regenerative purposes. Outer topography-modified scaffolds are those scaffolds whose outer surface are modified by nanopattern with controlled disorder, which could promote osteointegration and avoid the formation of fibrous tissue at the surface of bone implants (Dobbenga et al., 2016). For example, Silverwood et al. (2016) utilized a block-copolymer templated anodization technique to modify the outer surface of titania scaffolds with nanopillars (height $15 \mathrm{~nm}$ ) and then transplanted modified titania and polished titania to rabbit femurs. Compared with the flat titania, the outer topography-modified titania with $15 \mathrm{~nm}$ nanopillars show higher bone to implant contact (20\%) (Silverwood et al., 2016). Meanwhile, inter topographymodified scaffolds are those scaffolds whose porous inner surface are modified by controlled nanotopography. For example, Wang et al. used cold atmospheric plasma to treat or modify the inter topography of $3 \mathrm{D}$ printed poly-lactic-acid scaffolds, which could improve nanoscale surface roughness

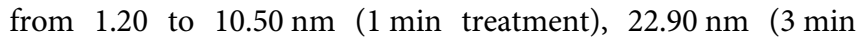
treatment), and $27.60 \mathrm{~nm}$ (5 min treatment) (Wang et al., 2016b). And modified scaffolds could improve cell adhesion and proliferation when compared with unmodified scaffolds, showing great potential for bone tissue engineering (Wang M. et al., 2016). Further studies should focus on the osteogenic effects of the modified inter surface, and related treating techniques should also be developed.

\subsubsection{Piezoelectric Scaffolds}

Natural bone is an electricity-generated tissue because of piezoelectric collagen ( $\mathrm{Yu}$ et al., 2017). Thus, piezoelectric scaffolds are developed to provide electric stimuli for bone regeneration, which include piezoelectric polymers such as PEDOT (Iandolo et al., 2016) and poly (vinylidene fluoride), piezoelectric ceramics such as $\mathrm{BaTiO}_{3}$ (Polley et al., 2020) and $\mathrm{K}_{0.5} \mathrm{Na}_{0.5} \mathrm{NbO}_{3}$ (Yu et al., 2017), and scaffolds containing piezoelectric particles such as piezoelectric ceramic particles (Mancuso et al., 2021) and nylon-11 nanoparticles (Ma et al., 2019)). When piezoelectric scaffolds are subjected to external mechanical stimuli (physiologically or additionally), electric stimuli will be induced for bone regeneration (Tang et al., 2017). Osteogenic differentiation can be obtained by high voltage output form piezoelectric scaffolds (Damaraju et al., 2017). However, the piezoelectricity of scaffolds should be optimized for practical applications. For example, Zhang et al. utilized annealing treatment to control $\beta$ phase contents, thus constructing piezoelectric poly (vinylidene fluoridetrifluoroethylene) $[\mathrm{P}(\mathrm{VDF}-\mathrm{TrFE})]$ membranes with different surface potentials $(-78$ and $-53 \mathrm{mV}$ ) (Zhang et al., 2018). When transplanted to critical calvarial defects of rats, all groups could promote bone regeneration, but $\mathrm{P}(\mathrm{VDF}-\mathrm{TrFE})$ membrane with $-53 \mathrm{mV}$ surface potential is better because it dramatically promotes faster bone regeneration with more mature bone structure than unpolarized group and that with $-78 \mathrm{mv}$ surface potential (Zhang et al., 2018). Furthermore, the osteoinductive effects of piezoelectric scaffolds can enhance additional mechanical stimuli. Piezoelectric scaffolds were fabricated by modifying porous Ti6Al4V scaffolds (pTi) with
$\mathrm{BaTiO}_{3}$, and then piezoelectric $\mathrm{pTi} / \mathrm{BaTiO}_{3}$ or pure pTi were transplanted to rabbit radius bone defects with a length of $13 \mathrm{~mm}$ (Fan et al., 2020). Results showed that $\mathrm{pTi} / \mathrm{BaTiO}_{3}$ with or without LIPUS promotes more bone regeneration than pure pTi, but LIPUS treatment could further enhance osteointegration and osteogenesis (Fan et al., 2020).

\subsubsection{Magnetically Actuated Scaffolds}

Magnetically actuated scaffolds are generally constructed by introducing magnetic particles (usually magnetite) to other biomaterial scaffolds (Santos et al., 2015). When magnetically actuated scaffolds are exposed in magnetic field, the deformation of scaffolds stimulates the differentiation of stem cells (Spangenberg et al., 2021). Aldebs et al. (2020) used PEGF $(1 \mathrm{mT}, 15 \mathrm{~Hz})$ to stimulate ADSCs for 7 days seeded in RADA16 self-assembling peptide hydrogel containing magnetic particles and found that PEGF coupled with magnetic particles could promote the osteogenic differentiation of ADSCs without spoiling cell viability. Moreover, magnetic nanoparticles can be encapsuled to piezoelectric scaffolds, which could provide magnetomechanical and electromagnetic stimuli (Fernandes et al., 2019). Zhang et al. fabricated magnetically actuated scaffolds with magnetic effects by simultaneously incorporating two magnetic particles: positive $\mathrm{CoFe}_{2} \mathrm{O}_{4}$ (CFO) and negative $\mathrm{Tb}_{\mathrm{x}} \mathrm{Dy}_{1-\mathrm{x}} \mathrm{Fe}_{2}$ alloy (TD) to piezoelectric P(VDFTrFE) (Zhang et al., 2020). A CFO/TD ratio of $4: 1$ could dramatically improve surface potential when scaffolds are exposed to magnetic field. Then, MSCs were seeded on the film for 7 or 14 days, and a magnetic field was applied at the $4^{\text {th }}$ or $8^{\text {th }}$ day. Results showed that magnetic field could dramatically stimulate the osteogenic differentiation of MSCs in the 14-days culture, but osteogenic differentiation was not observed at the 4-days culture, suggesting that magnetic fieldcoupled magnetic particles could promote osteogenesis, but sufficient exposure time is needed (Zhang et al., 2020). Although magnetically actuated scaffolds show satisfying biocompatibility when transplanted in vivo, additional evidence is needed about bone defect repair in vivo by magnetically actuated scaffolds coupled with magnetic field.

\subsection{Tissue Engineered Bone Grafts by in vitro Bioreactors}

In addition to prefabricated osteoinductive scaffolds by biophysical stimuli, they can be directly exerted by in vitro bioreactors to construct TEBGs, artificial autografts with anatomically matched shape and size (Grayson et al., 2010). TEBGs are generally fabricated by a combination of a biomaterial scaffold, autogenous stem cells, osteogenic supplements, and in vitro bioreactors providing biophysical stimuli (Fröhlich et al., 2010).

Among various in vitro bioreactors, load-induced FFSS-based bioreactors have been widely used to construct TEBGs, especially perfusion bioreactors. The efficiency of TEBGs for bone tissue engineering was initially verified by ectopic bone formation in animal models. Hosseinkhani et al. mixed MSC suspension with peptide amphiphile (PA) solution to form PA nanofiber hydrogel 
containing MSCs, which was then infiltrated to collagen/poly (glycolic acid) sponge for static or prefusion culture (Hosseinkhani et al., 2006). After 3 weeks of in vitro culture, both groups were transplanted to the back subcutis of rats for 8 weeks, and results showed that the TEBGs obtained by perfusion culture promote homogenous and robust bone regeneration when compared with those by static culture (Hosseinkhani et al., 2006). Moreover, TEBGs for critical bone defects have been verified in clinically relevant pig bone defect models. An image-guided personalized strategy was utilized to construct TEBGs by 3 weeks of invitro culture (Bhumiratana et al., 2016). TEBGs are constructed by a combination of decellularized bone matrixes (DBMs), autogenous ADSCs, osteogenic medium, and a perfusion-based bioreactor, and immure bone formation is observed after 3 weeks of perfusion culture. Then, TEBGs or acellular DBMs were transplanted to bone defects of mature Yucatán minipigs for 6 weeks, and results revealed that TEBGs show improved bone formation and vascularization compared with untreated defects and acellular scaffolds (Bhumiratana et al., 2016). Therefore, TEBGs show great promise for critical bone defects, which ideally integrate four pillars of bone tissue engineering.

TEBGs may be the second-generation autogenous bone grafts for surgical bone repair. Thus, various methods have been developed to improve the effectiveness of TEBGs, which are based on four hierarchies. The first level is to optimize perfusion profile, which includes perfusion duration and perfusion model. Dynamic culture for at least 2 weeks is needed to fabricate TEBGs (Mitra et al., 2017). In addition, sequential application of continuous perfusion and intermittent perfusion may be better than single model (Correia et al., 2013). The second level is to optimize bioreactor system medium. For example, marine hemoglobin could be incorporated to perfusion medium to deliver sufficient oxygen, which enhances the proliferation and osteogenic differentiation of MSCs in perfusion bioreactors (Le Pape et al., 2018). The third level is to optimize seed cells. For example, BMSCs may be superior to ADSCs because of better potential for osteogenesis (Wu et al., 2015). In addition, coculture of multiple cells may be superior to single cell culture for the fabrication of TEBGs (Sawyer et al., 2020), but multiple cells must be obtained from autogenous stem cells. Thus, co-culture of osteogenically indued stem cells and angiogenic-induced stem cells shows great promise. Furthermore, the fourth level is to optimize biomaterial scaffolds. Hydrogels can be used to modify other biomaterials because hydrogels could improve cell retention (Yu et al., 2012). In addition, bioactive molecules can be introduced to biomaterial scaffolds to provide sustained-release osteoinductive signaling (Panek et al., 2019).

In addition to perfusion bioreactors, load induced-FFSSbased bioreactors include rotating wall vessel bioreactors, spinner flask bioreactors, and biaxial rotating bioreactors, among which biaxial rotating bioreactors may surpass other uni-axial perfusion bioreactors because they generate homogenous FFSS and promote robust osteogenesis (Singh et al., 2005; Zhang et al., 2010a). TEBGs fabricated by biaxial rotating bioreactors induce ectopic bone regeneration (Zhang et al., 2009) and promote the healing of critical femoral defects of rats (Zhang et al., 2010b).

Other bioreactors include compression bioreactors (Ravichandran et al., 2017), tension bioreactors (Carroll et al., 2017), nanovibration bioreactors (Wu et al., 2020), ultrasound bioreactors (Wang M. et al., 2016), and electromagnetic bioreactors (Fernandes et al., 2019), which show promise to construct TEBGS, but they are limited to transport nitrogen, oxygen, and growth factors. Therefore, other bioreactors can be synthetically used with load induce-FFSS based bioreactors to design multi-biophysical stimuli bioreactors. For example, a multimodal bioreactor was designed which could provide compression and load-induced FFSS, and osteogenic differentiation is improved when compared with static culture and culture with single biophysical stimuli when the system is used to dynamically culture MSCs (Ravichandran et al., 2018). Further evidence is needed about TEBGs fabricated by multimodal bioreactors to repair critical bone defects.

\subsection{Postoperative Biophysical Stimuli Loading Strategies}

Postoperative biophysical stimuli loading strategies are those methods that use noninvasive methods to generate biophysical stimuli to stimulate bone regeneration after surgery, including distraction osteogenesis (DO) (Shah et al., 2021), LMHFV (Steppe et al., 2020), LIPUS (Hannemann et al., 2014), and PEMF (Wang et al., 2019). These strategies have been clinically used to promote the healing of fractures; thus, their use can be extended to the treatment of critical bone defects. For example, Parmaksiz et al. transplanted DBM and DBM containing magnetic particles to bilateral critical-size cranial defects of rats with or without PEMF exposure, and results revealed that exposed groups of DBM and DBM containing magnetic particles show improved bone regeneration and reduced fibrous formation compared with unexposed groups (Parmaksiz et al., 2021). Yan et al. also verified that postoperative LIPUS could promote the healing of rabbit femurs defects (Yan et al., 2016). Therefore, postoperative biophysical stimuli loading strategies show great promise in bone tissue engineering, but they remain poorly explored. Bone defects are usually limited to local regions. Thus, small loading devices are also highly developed for clinical bone repair. When postoperative biophysical stimuli loading strategies are used in combination of other pillars of bone tissue engineering, their clinical complications or drawbacks may diminish. For example, DO is a long-term treatment that may cause infections or delay, but it shows great promise for bone regeneration when combined with osteoinducive $\mathrm{Mg}$ nail, which could reduce the treatment time of traditional DO (Ye et al., 2021).

\section{DISCUSSION}

Considering the inefficiency of current biomaterials and bioactive molecules, biophysical stimuli are critical to be applied as the 
fourth pillar of bone tissue engineering. In addition, biophysical stimuli should not be only limited to external mechanical stimuli, but in a more comprehensive concept, the fourth pillar can also include internal structural stimuli, acoustic stimuli, and electromagnetic stimuli. Although distinctive biophysical stimuli are based on different physical properties, they will be transferred to mechanical or electromagnetic stimuli. Given that both show reminiscent signal pathways for MSC osteogenesis, a novel concept of biophysical transduction is proposed to incorporate mechanotransduction and electrocoupling to interpret the osteoinductive mechanisms of biophysical stimuli for osteogenic differentiation. Biophysical transduction can be divided into three stages: sensing, transmission, and regulation. Depending on sensing pattern, biophysical transduction can be categorized into self-biophysical transduction, cell-matrix biophysical transduction, and cell-cell biophysical transduction. Furthermore, biophysical stimuli, as the fourth pillar of bone tissue engineering, can be used by fabricating preconstructed scaffolds with osteoinductive properties and TEBGs or by employing postoperative biophysical stimuli loading strategies.

While biophysical stimuli show promising potential to be used as the fourth pillar of bone tissue engineering, ideal biophysical stimuli are needed to promote cell recruitment, proliferation, osteogenesis, angiogenesis, neurogenesis, and immune regulation. Osteoinductive windows of biophysical stimuli need to be comprehensively considered and optimized. The synergy effects of multiple biophysical stimuli should be also explored for bone regeneration. Moreover, specific osteoinductive mechanisms of biophysical stimuli need further investigation. The interaction between mechanotransduction and electrocoupling should be probed to interpret their similarity. Whether or not other cell adhesion receptors and ligands

\section{REFERENCES}

Al Nazer, R., Lanovaz, J., Kawalilak, C., Johnston, J. D., and Kontulainen, S. (2012). Direct In Vivo Strain Measurements in Human Bone-A Systematic Literature Review. J. Biomech. 45, 27-40. doi:10.1016/ j.jbiomech.2011.08.004

Aldebs, A. I., Zohora, F. T., Nosoudi, N., Singh, S. P., and Ramirez-Vick, J. E. (2020). Effect of Pulsed Electromagnetic fields on Human Mesenchymal Stem Cells Using 3d Magnetic Scaffolds. Bioelectromagnetics 41, 175-187. doi:10.1002/bem. 22248

Arjmand, M., Ardeshirylajimi, A., Maghsoudi, H., and Azadian, E. (2018). Osteogenic Differentiation Potential of Mesenchymal Stem Cells Cultured on Nanofibrous Scaffold Improved in the Presence of Pulsed Electromagnetic Field. J. Cell Physiol 233, 1061-1070. doi:10.1002/jcp.25962

Arnsdorf, E. J., Tummala, P., and Jacobs, C. R. (2009a). Non-Canonical Wnt Signaling and N-Cadherin Related $\beta$-Catenin Signaling Play a Role in Mechanically Induced Osteogenic Cell Fate. PloS one 4, e5388. doi:10.1371/ journal.pone.0005388

Arnsdorf, E. J., Tummala, P., Kwon, R. Y., and Jacobs, C. R. (2009b). Mechanically Induced Osteogenic Differentiation - the Role of RhoA, ROCKII and Cytoskeletal Dynamics. J. Cel. Sci. 122, 546-553. doi:10.1242/jcs.036293

Aziz, A. H., Eckstein, K., Ferguson, V. L., and Bryant, S. J. (2019). The Effects of Dynamic Compressive Loading on Human Mesenchymal Stem Cell Osteogenesis in the Stiff Layer of a Bilayer Hydrogel. J. Tissue Eng. Regen. Med. 13, 946-959. doi:10.1002/term.2827 participate in cell-matrix biophysical transduction remains unknown. The autocrine and paracrine network of cell-cell biophysical transduction also needs to be refined. In vivo preclinical evidence about preconstructed scaffolds may be obtained, especially for piezoelectric scaffolds and magnetically actuated scaffolds. Novel in vitro bioreactors may be designed to diminish complexity and volume. Further studies may focus on the application of postoperative biophysical stimuli loading strategies for the treatment of critical bone defects. Specific parameters using model and duration may be established.

\section{AUTHOR CONTRIBUTIONS}

$\mathrm{ZH}, \mathrm{ZX}$, and XW contributed equally to this work. $\mathrm{ZH}$ researched and analyzed the literature, prepared the manuscript, and drew the figures. ZX and XW researched and analyzed the literature, prepared the manuscript. HL and TC researched the literature and modified the manuscript. $\mathrm{YH}$, $\mathrm{YW}, \mathrm{RC}$ and $\mathrm{KH}$ modified the manuscript and figures. JL and CC supervised, administrated and edited the work. All authors have approved for the publication.

\section{FUNDING}

We thanks to the funds to support the work, which include the National Natural Science Foundation of China (No: 81871752), Hubei Provincial Natural Science Foundation (No: 2020CFB551), Zhongnan Hospital of Wuhan University Science, Technology and Innovation Seed Fund (No: cxpy2019074), and Translational Medicine and Interdisciplinary Research Joint Fund of Zhongnan Hospital of Wuhan University (No. ZNJC202014).

Bagheri, L., Pellati, A., Rizzo, P., Aquila, G., Massari, L., De Mattei, M., et al. (2018). Notch Pathway Is Active during Osteogenic Differentiation of Human Bone Marrow Mesenchymal Stem Cells Induced by Pulsed Electromagnetic fields. J. Tissue Eng. Regen. Med. 12, 304-315. doi:10.1002/term.2455

Baumgartner, W., Schneider, I., Hess, S. C., Stark, W. J., Märsmann, S., Brunelli, M., et al. (2018). Cyclic Uniaxial Compression of Human Stem Cells Seeded on a Bone Biomimetic Nanocomposite Decreases Anti-osteogenic Commitment Evoked by Shear Stress. J. Mech. Behav. Biomed. Mater. 83, 84-93. doi:10.1016/j.jmbbm.2018.04.002

Benayahu, D., Wiesenfeld, Y., and Sapir-Koren, R. (2019). How Is Mechanobiology Involved in Mesenchymal Stem Cell Differentiation toward the Osteoblastic or Adipogenic Fate? J. Cell Physiol 234, 12133-12141. doi:10.1002/jcp.28099

Bertrand, A. A., Malapati, S. H., Yamaguchi, D. T., and Lee, J. C. (2020). The Intersection of Mechanotransduction and Regenerative Osteogenic Materials. Adv. Healthc. Mater. 9, 2000709. doi:10.1002/adhm.202000709

Bhavsar, M. B., Cato, G., Hauschild, A., Leppik, L., Costa Oliveira, K. M., EischenLoges, M. J., et al. (2019). Membrane Potential (Vmem) Measurements during Mesenchymal Stem Cell (MSC) Proliferation and Osteogenic Differentiation. PeerJ 7, e6341. doi:10.7717/peerj.6341

Bhumiratana, S., Bernhard, J. C., Alfi, D. M., Yeager, K., Eton, R. E., Bova, J., et al. (2016). Tissue-engineered Autologous Grafts for Facial Bone Reconstruction. Sci. Transl. Med. 8, 343ra83. doi:10.1126/scitranslmed.aad5904

Birks, S., and Uzer, G. (2021). At the Nuclear Envelope of Bone Mechanobiology. Bone 151, 116023. doi:10.1016/j.bone.2021.116023

Bjerre, L., Bünger, C. E., Kassem, M., and Mygind, T. (2008). Flow Perfusion Culture of Human Mesenchymal Stem Cells on Silicate-Substituted Tricalcium 
Phosphate Scaffolds. Biomaterials 29, 2616-2627. doi:10.1016/ j.biomaterials.2008.03.003

Boda, S. K., Thrivikraman, G., and Basu, B. (2015). Magnetic Field Assisted Stem Cell Differentiation - Role of Substrate Magnetization in Osteogenesis. J. Mater. Chem. B 3, 3150-3168. doi:10.1039/c5tb00118h

Bouzid, T., Kim, E., Riehl, B. D., Esfahani, A. M., Rosenbohm, J., Yang, R., et al. (2019). The Linc Complex, Mechanotransduction, and Mesenchymal Stem Cell Function and Fate. J. Biol. Eng. 13, 68. doi:10.1186/s13036-019-0197-9

Brady, R. T., O'Brien, F. J., and Hoey, D. A. (2015). Mechanically Stimulated Bone Cells Secrete Paracrine Factors that Regulate Osteoprogenitor Recruitment, Proliferation, and Differentiation. Biochem. biophysical Res. Commun. 459, 118-123. doi:10.1016/j.bbrc.2015.02.080

Burks, S. R., Nagle, M. E., Bresler, M. N., Kim, S. J., Star, R. A., and Frank, J. A. (2018). Mesenchymal Stromal Cell Potency to Treat Acute Kidney Injury Increased by Ultrasound-Activated Interferon-Г/interleukin-10 axis. J. Cell Mol Med 22, 6015-6025. doi:10.1111/jcmm.13874

Carroll, S. F., Buckley, C. T., and Kelly, D. J. (2017). Cyclic Tensile Strain Can Play a Role in Directing Both Intramembranous and Endochondral Ossification of Mesenchymal Stem Cells. Front. Bioeng. Biotechnol. 5, 73. doi:10.3389/ fbioe. 2017.00073

Case, N., Thomas, J., Xie, Z., Sen, B., Styner, M., Rowe, D., et al. (2013). Mechanical Input Restrains PPAR $\gamma 2$ Expression and Action to Preserve Mesenchymal Stem Cell Multipotentiality. Bone 52, 454-464. doi:10.1016/j.bone.2012.08.122

Cashion, A. T., Caballero, M., Halevi, A., Pappa, A., Dennis, R. G., and van Aalst, J. A. (2014). Programmable Mechanobioreactor for Exploration of the Effects of Periodic Vibratory Stimulus on Mesenchymal Stem Cell Differentiation. BioResearch open access 3, 19-28. doi:10.1089/biores.2013.0048

Chang, Y., Shao, Y., Liu, Y., Xia, R., Tong, Z., Zhang, J., et al. (2019). Mechanical Strain Promotes Osteogenic Differentiation of Mesenchymal Stem Cells on TiO2 Nanotubes Substrate. Biochem. biophysical Res. Commun. 511, 840-846. doi:10.1016/j.bbrc.2019.02.145

Chen, J. C., Hoey, D. A., Chua, M., Bellon, R., and Jacobs, C. R. (2016). Mechanical Signals Promote Osteogenic Fate through a Primary Cilia-mediated Mechanism. FASEB j. 30, 1504-1511. doi:10.1096/fj.15-276402

Chen, J., Tu, C., Tang, X., Li, H., Yan, J., Ma, Y., et al. (2019). The Combinatory Effect of Sinusoidal Electromagnetic Field and Vegf Promotes Osteogenesis and Angiogenesis of Mesenchymal Stem Cell-Laden Pcl/ha Implants in a Rat Subcritical Cranial Defect. Stem Cell Res Ther 10, 379. doi:10.1186/s13287019-1464-x

Chen, X., He, F., Zhong, D.-Y., and Luo, Z.-P. (20152015). Acoustic-frequency Vibratory Stimulation Regulates the Balance between Osteogenesis and Adipogenesis of Human Bone Marrow-Derived Mesenchymal Stem Cells. Biomed. Research International 2015, 1-10. doi:10.1155/2015/540731

Cho, M. R., Thatte, H. S., Silvia, M. T., and Golan, D. E. (1999). Transmembrane Calcium Influx Induced by Ac Electric fields. FASEB j. 13, 677-683. doi:10.1096/fasebj.13.6.677

Correia, C., Bhumiratana, S., Sousa, R. A., Reis, R. L., and Vunjak-Novakovic, G. (2013). Sequential Application of Steady and Pulsatile Medium Perfusion Enhanced the Formation of Engineered Bone. Tissue Eng. A 19, 1244-1254. doi:10.1089/ten.TEA.2011.0701

Corrigan, M. A., Johnson, G. P., Stavenschi, E., Riffault, M., Labour, M.-N., and Hoey, D. A. (2018). Trpv4-mediates Oscillatory Fluid Shear Mechanotransduction in Mesenchymal Stem Cells in Part via the Primary Cilium. Sci. Rep. 8, 3824. doi:10.1038/s41598-018-22174-3

Creecy, C. M., O'Neill, C. F., Arulanandam, B. P., Sylvia, V. L., Navara, C. S., and Bizios, R. (2013). Mesenchymal Stem Cell Osteodifferentiation in Response to Alternating Electric Current. Tissue Eng. Part A 19, 467-474. doi:10.1089/ ten.TEA.2012.0091

Dalby, M. J., Gadegaard, N., and Oreffo, R. O. C. (2014). Harnessing Nanotopography and Integrin-Matrix Interactions to Influence Stem Cell Fate. Nat. Mater 13, 558-569. doi:10.1038/nmat3980

Dalby, M. J., Gadegaard, N., Tare, R., Andar, A., Riehle, M. O., Herzyk, P., et al. (2007). The Control of Human Mesenchymal Cell Differentiation Using Nanoscale Symmetry and Disorder. Nat. Mater 6, 997-1003. doi:10.1038/ nmat2013

Damaraju, S. M., Shen, Y., Elele, E., Khusid, B., Eshghinejad, A., Li, J., et al. (2017). Three-dimensional Piezoelectric Fibrous Scaffolds Selectively Promote
Mesenchymal Stem Cell Differentiation. Biomaterials 149, 51-62. doi:10.1016/j.biomaterials.2017.09.024

Dash, S. K., Sharma, V., Verma, R. S., and Das, S. K. (2020). Low Intermittent Flow Promotes Rat Mesenchymal Stem Cell Differentiation in Logarithmic Fluid Shear Device. Biomicrofluidics 14, 054107. doi:10.1063/5.0024437

Datta, N., Pham, Q. P., Sharma, U., Sikavitsas, V. I., Jansen, J. A., and Mikos, A. G. (2006). In Vitro generated Extracellular Matrix and Fluid Shear Stress Synergistically Enhance 3d Osteoblastic Differentiation. Proc. Natl. Acad. Sci. 103, 2488-2493. doi:10.1073/pnas.0505661103

de Lucas, B., Pérez, L. M., Bernal, A., and Gálvez, B. G. (2020). Ultrasound Therapy: Experiences and Perspectives for Regenerative Medicine. Genes 11, 1086. doi:10.3390/genes11091086

Delaine-Smith, R. M., and Reilly, G. C. (2012). Mesenchymal Stem Cell Responses to Mechanical Stimuli. Muscles Ligaments Tendons J. 2, 169-180.

Ding, X., Zhao, H., Li, Y., Lee, A. L., Li, Z., Fu, M., et al. (2020). Synthetic Peptide Hydrogels as 3d Scaffolds for Tissue Engineering. Adv. Drug Deliv. Rev. 160, 78-104. doi:10.1016/j.addr.2020.10.005

Dobbenga, S., Fratila-Apachitei, L. E., and Zadpoor, A. A. (2016). Nanopatterninduced Osteogenic Differentiation of Stem Cells - a Systematic Review. Acta Biomater. 46, 3-14. doi:10.1016/j.actbio.2016.09.031

Dong, L., Song, Y., Zhang, Y., Zhao, W., Wang, C., Lin, H., et al. (2021). Mechanical Stretch Induces Osteogenesis through the Alternative Activation of Macrophages. J. Cell Physiol 236, 6376-6390. doi:10.1002/jcp.30312

Driscoll, T. P., Cosgrove, B. D., Heo, S.-J., Shurden, Z. E., and Mauck, R. L. (2015). Cytoskeletal to Nuclear Strain Transfer Regulates Yap Signaling in Mesenchymal Stem Cells. Biophysical J. 108, 2783-2793. doi:10.1016/ j.bpj.2015.05.010

Dupont, S., Morsut, L., Aragona, M., Enzo, E., Giulitti, S., Cordenonsi, M., et al. (2011). Role of Yap/taz in Mechanotransduction. Nature 474, 179-183. doi:10.1038/nature10137

Eischen-Loges, M., Oliveira, K. M. C., Bhavsar, M. B., Barker, J. H., and Leppik, L. (2018). Pretreating Mesenchymal Stem Cells with Electrical Stimulation Causes Sustained Long-Lasting Pro-osteogenic Effects. PeerJ 6, e4959. doi:10.7717/ peer. 4959

Elosegui-Artola, A., Andreu, I., Beedle, A. E. M., Lezamiz, A., Uroz, M., Kosmalska, A. J., et al. (2017). Force Triggers Yap Nuclear Entry by Regulating Transport across Nuclear Pores. Cell 171, 1397-1410. e14. doi:10.1016/j.cell.2017.10.008

Engler, A. J., Sen, S., Sweeney, H. L., and Discher, D. E. (2006). Matrix Elasticity Directs Stem Cell Lineage Specification. Cell 126, 677-689. doi:10.1016/ j.cell.2006.06.044

Esfandiari, E., Roshankhah, S., Mardani, M., Hashemibeni, B., Naghsh, E., Kazemi, M., et al. (2014). The Effect of High Frequency Electric Field on Enhancement of Chondrogenesis in Human Adipose-Derived Stem Cells. Iran J. Basic Med. Sci. $17,571-576$

Esposito, M., Lucariello, A., Riccio, I., Riccio, V., Esposito, V., and Riccardi, G. (2012). Differentiation of Human Osteoprogenitor Cells Increases after Treatment with Pulsed Electromagnetic fields. In Vivo 26, 299-304.

Fan, B., Guo, Z., Li, X., Li, S., Gao, P., Xiao, X., et al. (2020). Electroactive Barium Titanate Coated Titanium Scaffold Improves Osteogenesis and Osseointegration with Low-Intensity Pulsed Ultrasound for Large Segmental Bone Defects. Bioactive Mater. 5, 1087-1101. doi:10.1016/ j.bioactmat.2020.07.001

Fernandes, M. M., Correia, D. M., Ribeiro, C., Castro, N., Correia, V., and Lanceros-Mendez, S. (2019). Bioinspired Three-Dimensional Magnetoactive Scaffolds for Bone Tissue Engineering. ACS Appl. Mater. Inter. 11, 45265-45275. doi:10.1021/acsami.9b14001

Filipowska, J., Reilly, G. C., and Osyczka, A. M. (2016). A Single Short Session of media Perfusion Induces Osteogenesis in Hbmscs Cultured in Porous Scaffolds, Dependent on Cell Differentiation Stage. Biotechnol. Bioeng. 113, 1814-1824. doi:10.1002/bit.25937

Fröhlich, M., Grayson, W. L., Marolt, D., Gimble, J. M., Kregar-Velikonja, N., and Vunjak-Novakovic, G. (2010). Bone Grafts Engineered from Human AdiposeDerived Stem Cells in Perfusion Bioreactor Culture. Tissue Eng. Part A 16, 179-189. doi:10.1089/ten.TEA.2009.0164

Fu, J., Liu, X., Tan, L., Cui, Z., Liang, Y., Li, Z., et al. (2020). Modulation of the Mechanosensing of Mesenchymal Stem Cells by Laser-Induced Patterning for the Acceleration of Tissue Reconstruction through the Wnt/ $\beta$-Catenin 
Signaling Pathway Activation. Acta Biomater. 101, 152-167. doi:10.1016/ j.actbio.2019.10.041

Gharibi, B., Cama, G., Capurro, M., Thompson, I., Deb, S., Di Silvio, L., et al. (2013). Gene Expression Responses to Mechanical Stimulation of Mesenchymal Stem Cells Seeded on Calcium Phosphate Cement. Tissue Eng. Part A 19, 2426-2438. doi:10.1089/ten.tea.2012.0623

Glassman, M. J., Avery, R. K., Khademhosseini, A., and Olsen, B. D. (2016). Toughening of Thermoresponsive Arrested Networks of Elastin-like Polypeptides to Engineer Cytocompatible Tissue Scaffolds. Biomacromolecules 17, 415-426. doi:10.1021/acs.biomac.5b01210

Grayson, W. L., Fröhlich, M., Yeager, K., Bhumiratana, S., Chan, M. E., Cannizzaro, C., et al. (2010). Engineering Anatomically Shaped Human Bone Grafts. Proc. Natl. Acad. Sci. USA 107, 3299-3304. doi:10.1073/pnas.0905439106

Haffner-Luntzer, M., Liedert, A., and Ignatius, A. (2016). Mechanobiology of Bone Remodeling and Fracture Healing in the Aged Organism. Innovative Surg. Sci. 1, 57-63. doi:10.1515/iss-2016-0021

Hannemann, P. F. W., Mommers, E. H. H., Schots, J. P. M., Brink, P. R. G., and Poeze, M. (2014). The Effects of Low-Intensity Pulsed Ultrasound and Pulsed Electromagnetic fields Bone Growth Stimulation in Acute Fractures: A Systematic Review and Meta-Analysis of Randomized Controlled Trials. Arch. Orthop. Trauma Surg. 134, 1093-1106. doi:10.1007/s00402-014-2014-8

Hao, J., Zhang, Y., Jing, D., Shen, Y., Tang, G., Huang, S., et al. (2015). Mechanobiology of Mesenchymal Stem Cells: Perspective into Mechanical Induction of Msc Fate. Acta Biomater. 20, 1-9. doi:10.1016/j.actbio.2015.04.008

Haudenschild, A. K., Hsieh, A. H., Kapila, S., and Lotz, J. C. (2009). Pressure and Distortion Regulate Human Mesenchymal Stem Cell Gene Expression. Ann. Biomed. Eng. 37, 492-502. doi:10.1007/s10439-008-9629-2

Hayakawa, K., Tatsumi, H., and Sokabe, M. (2011). Actin Filaments Function as a Tension Sensor by Tension-dependent Binding of Cofilin to the Filament. J. Cel. Biol. 195, 721-727. doi:10.1083/jcb.201102039

Henstock, J. R., Rotherham, M., Rashidi, H., Shakesheff, K. M., and El Haj, A. J. (2014). Remotely Activated Mechanotransduction via Magnetic Nanoparticles Promotes Mineralization Synergistically with Bone Morphogenetic Protein 2: Applications for Injectable Cell Therapy. Stem Cell translational Med. 3, 1363-1374. doi:10.5966/sctm.2014-0017

Hess, R., Jaeschke, A., Neubert, H., Hintze, V., Moeller, S., Schnabelrauch, M., et al. (2012). Synergistic Effect of Defined Artificial Extracellular Matrices and Pulsed Electric fields on Osteogenic Differentiation of Human Mscs. Biomaterials 33, 8975-8985. doi:10.1016/j.biomaterials.2012.08.056

Hoey, D. A., Kelly, D. J., and Jacobs, C. R. (2011). A Role for the Primary Cilium in Paracrine Signaling between Mechanically Stimulated Osteocytes and Mesenchymal Stem Cells. Biochem. biophysical Res. Commun. 412, 182-187. doi:10.1016/j.bbrc.2011.07.072

Hoey, D. A., Tormey, S., Ramcharan, S., O’Brien, F. J., and Jacobs, C. R. (2012). Primary Cilia-Mediated Mechanotransduction in Human Mesenchymal Stem Cells. Stem Cells 30, 2561-2570. doi:10.1002/stem.1235

Horner, C. B., Hirota, K., Liu, J., Maldonado, M., Hyle Park, B., and Nam, J. (2018). Magnitude-dependent and Inversely-related Osteogenic/chondrogenic Differentiation of Human Mesenchymal Stem Cells under Dynamic Compressive Strain. J. Tissue Eng. Regen. Med. 12, e637-e647. doi:10.1002/ term. 2332

Hosseinkhani, H., Hosseinkhani, M., Tian, F., Kobayashi, H., and Tabata, Y. (2006). Ectopic Bone Formation in Collagen Sponge Self-Assembled PeptideAmphiphile Nanofibers Hybrid Scaffold in a Perfusion Culture Bioreactor. Biomaterials 27, 5089-5098. doi:10.1016/j.biomaterials.2006.05.050

Hronik-Tupaj, M., Rice, W. L., Cronin-Golomb, M., Kaplan, D. L., and Georgakoudi, I. (2011). Osteoblastic Differentiation and Stress Response of Human Mesenchymal Stem Cells Exposed to Alternating Current Electric fields. Biomed. Eng. Online 10, 9. doi:10.1186/1475-925x-10-9

Hu, B., Haj, A., and Dobson, J. (2013). Receptor-targeted, Magneto-Mechanical Stimulation of Osteogenic Differentiation of Human Bone Marrow-Derived Mesenchymal Stem Cells. Ijms 14, 19276-19293. doi:10.3390/ijms140919276

Hu, B., Zhang, Y., Zhou, J., Li, J., Deng, F., Wang, Z., et al. (2014). Low-intensity Pulsed Ultrasound Stimulation Facilitates Osteogenic Differentiation of Human Periodontal Ligament Cells. PloS one 9, e95168. doi:10.1371/ journal.pone. 0095168

Hu, Z., Zhang, L., Wang, H., Wang, Y., Tan, Y., Dang, L., et al. (2020). Targeted Silencing of Mirna-132-3p Expression Rescues Disuse Osteopenia by
Promoting Mesenchymal Stem Cell Osteogenic Differentiation and Osteogenesis in Mice. Stem Cell Res Ther 11, 58. doi:10.1186/s13287-0201581-6

Huang, C., and Ogawa, R. (2012). Effect of Hydrostatic Pressure on Bone Regeneration Using Human Mesenchymal Stem Cells. Tissue Eng. Part A 18, 2106-2113. doi:10.1089/ten.TEA.2012.0064

Huang, J., Lin, D., Wei, Z., Li, Q., Zheng, J., Zheng, Q., et al. (2020). Parathyroid Hormone Derivative with Reduced Osteoclastic Activity Promoted Bone Regeneration via Synergistic Bone Remodeling and Angiogenesis. Small 16, 1905876. doi:10.1002/smll.201905876

Huebsch, N., Arany, P. R., Mao, A. S., Shvartsman, D., Ali, O. A., Bencherif, S. A., et al. (2010). Harnessing Traction-Mediated Manipulation of the Cell/matrix Interface to Control Stem-Cell Fate. Nat. Mater 9, 518-526. doi:10.1038/ nmat 2732

Iandolo, D., Ravichandran, A., Liu, X., Wen, F., Chan, J. K. Y., Berggren, M., et al. (2016). Development and Characterization of Organic Electronic Scaffolds for Bone Tissue Engineering. Adv. Healthc. Mater. 5, 1505-1512. doi:10.1002/ adhm. 201500874

Jazayeri, M., Shokrgozar, M. A., Haghighipour, N., Bolouri, B., Mirahmadi, F., and Farokhi, M. (2017). Effects of Electromagnetic Stimulation on Gene Expression of Mesenchymal Stem Cells and Repair of Bone Lesions. Cell J 19, 34-44. doi:10.22074/celli.2016.4870

Johnson, G. P., Fair, S., and Hoey, D. A. (2021). Primary Cilium-Mediated Msc Mechanotransduction Is Dependent on Gpr161 Regulation of Hedgehog Signalling. Bone 145, 115846. doi:10.1016/j.bone.2021.115846

Johnson, G. P., Stavenschi, E., Eichholz, K. F., Corrigan, M. A., Fair, S., and Hoey, D. A. (2018). Mesenchymal Stem Cell Mechanotransduction Is Camp Dependent and Regulated by Adenylyl Cyclase 6 and the Primary Cilium. J. Cel. Sci. 131. doi:10.1242/jcs. 222737

Kanczler, J. M., Sura, H. S., Magnay, J., Green, D., Oreffo, R. O. C., Dobson, J. P., et al. (2010). Controlled Differentiation of Human Bone Marrow Stromal Cells Using Magnetic Nanoparticle Technology. Tissue Eng. Part A 16, 3241-3250. doi:10.1089/ten.TEA.2009.0638

Kapat, K., Shubhra, Q. T. H., Zhou, M., and Leeuwenburgh, S. (2020). Piezoelectric Nano-Biomaterials for Biomedicine and Tissue Regeneration. Adv. Funct. Mater. 30, 1909045. doi:10.1002/adfm.201909045

Kearney, E. M., Farrell, E., Prendergast, P. J., and Campbell, V. A. (2010). Tensile Strain as a Regulator of Mesenchymal Stem Cell Osteogenesis. Ann. Biomed. Eng. 38, 1767-1779. doi:10.1007/s10439-010-9979-4

Khare, D., Basu, B., and Dubey, A. K. (2020). Electrical Stimulation and Piezoelectric Biomaterials for Bone Tissue Engineering Applications. Biomaterials 258, 120280. doi:10.1016/j.biomaterials.2020.120280

Khaw, J. S., Xue, R., Cassidy, N. J., and Cartmell, S. H. (2021). Electrical Stimulation of Titanium to Promote Stem Cell Orientation, Elongation and Osteogenesis. Acta Biomater., S1742. doi:10.1016/ j.actbio.2021.08.010

Kim, K. M., Choi, Y. J., Hwang, J.-H., Kim, A. R., Cho, H. J., Hwang, E. S., et al. (2014). Shear Stress Induced by an Interstitial Level of Slow Flow Increases the Osteogenic Differentiation of Mesenchymal Stem Cells through Taz Activation. PloS one 9, e92427. doi:10.1371/journal.pone.0092427

Krishnan, L., Priddy, L. B., Esancy, C., Klosterhoff, B. S., Stevens, H. Y., Tran, L., et al. (2017). Delivery Vehicle Effects on Bone Regeneration and Heterotopic Ossification Induced by High Dose Bmp-2. Acta Biomater. 49, 101-112. doi:10.1016/j.actbio.2016.12.012

Labusca, L., Herea, D.-D., Danceanu, C.-M., Minuti, A. E., Stavila, C., Grigoras, M., et al. (2020). The Effect of Magnetic Field Exposure on Differentiation of Magnetite Nanoparticle-Loaded Adipose-Derived Stem Cells. Mater. Sci. Eng. C 109, 110652. doi:10.1016/j.msec.2020.110652

Lau, E., Lee, W. D., Li, J., Xiao, A., Davies, J. E., Wu, Q., et al. (2011). Effect of LowMagnitude, High-Frequency Vibration on Osteogenic Differentiation of Rat Mesenchymal Stromal Cells. J. Orthop. Res. 29, 1075-1080. doi:10.1002/ jor.21334

Lavenus, S., Berreur, M., Berreur, M., Trichet, V., Pilet, P., Louarn, G., et al. (2011). Adhesion and Osteogenic Differentiation of Human Mesenchymal Stem Cells on Titanium Nanopores. eCM 22, 84-96. doi:10.22203/ecm.v022a 07

Le Pape, F., Richard, G., Porchet, E., Sourice, S., Dubrana, F., Férec, C., et al. (2018). Adhesion, Proliferation and Osteogenic Differentiation of Human Mscs Cultured under Perfusion with a marine Oxygen Carrier on an Allogenic 
Bone Substitute. Artif. Cell nanomedicine, Biotechnol. 46, 95-107. doi:10.1080/ 21691401.2017.1365724

Leckband, D. E., and de Rooij, J. (2014). Cadherin Adhesion and Mechanotransduction. Annu. Rev. Cell Dev. Biol. 30, 291-315. doi:10.1146/ annurev-cellbio-100913-013212

Lee, J.-M., Kim, M.-G., Byun, J.-H., Kim, G.-C., Ro, J.-H., Hwang, D.-S., et al. (2017). The Effect of Biomechanical Stimulation on Osteoblast Differentiation of Human Jaw Periosteum-Derived Stem Cells. Maxillofac. Plast. Reconstr. Surg. 39, 7. doi:10.1186/s40902-017-0104-6

Lee, J. S., Ha, L., Park, J.-H., and Lim, J. Y. (2012). Mechanical Stretch Suppresses Bmp4 Induction of Stem Cell Adipogenesis via Upregulating Erk but Not through Downregulating Smad or P38. Biochem. biophysical Res. Commun. 418, 278-283. doi:10.1016/j.bbrc.2012.01.010

Li, C. W., Lau, Y. T., Lam, K. L., and Chan, B. P. (2020). Mechanically Induced Formation and Maturation of 3d-Matrix Adhesions (3dmas) in Human Mesenchymal Stem Cells. Biomaterials 258, 120292. doi:10.1016/ j.biomaterials.2020.120292

Li, R., Liang, L., Dou, Y., Huang, Z., Mo, H., Wang, Y., et al. (2015a). Mechanical Strain Regulates Osteogenic and Adipogenic Differentiation of Bone Marrow Mesenchymal Stem Cells. Biomed. Research International 2015, 1-10. doi:10.1155/2015/873251

Li, R., Liang, L., Dou, Y., Huang, Z., Mo, H., Wang, Y., et al. (2015b). Mechanical Stretch Inhibits Mesenchymal Stem Cell Adipogenic Differentiation through TGF $31 /$ Smad2 Signaling. J. Biomech. 48, 3656-3662. doi:10.1016/ j.jbiomech.2015.08.013

Lim, K., Hexiu, J., Kim, J., Seonwoo, H., Cho, W. J., Choung, P.-H., et al. (2013). Effects of Electromagnetic fields on Osteogenesis of Human Alveolar BoneDerived Mesenchymal Stem Cells. Biomed. Research International 2013, 1-14. doi:10.1155/2013/296019

Liu, L., Shao, L., Li, B., Zong, C., Li, J., Zheng, Q., et al. (2011). Extracellular SignalRegulated Kinase1/2 Activated by Fluid Shear Stress Promotes Osteogenic Differentiation of Human Bone Marrow-Derived Mesenchymal Stem Cells through Novel Signaling Pathways. Int. J. Biochem. Cell Biol. 43, 1591-1601. doi:10.1016/j.biocel.2011.07.008

Liu, L., Zong, C., Li, B., Shen, D., Tang, Z., Chen, J., et al. (2014). The Interaction Between $\beta 1$ Integrins and ERK1/2 in Osteogenic Differentiation of Human Mesenchymal Stem Cells under Fluid Shear Stress Modelled by a Perfusion System. J. Tissue Eng. Regen. Med. 8, 85-96. doi:10.1002/term.1498

Lohberger, B., Kaltenegger, H., Stuendl, N., Payer, M., Rinner, B., and Leithner, A. (20142014). Effect of Cyclic Mechanical Stimulation on the Expression of Osteogenesis Genes in Human Intraoral Mesenchymal Stromal and Progenitor Cells. Biomed. Research International 2014, 1-10. doi:10.1155/2014/189516

Lopes, D., Martins-Cruz, C., Oliveira, M. B., and Mano, J. F. (2018). Bone Physiology as Inspiration for Tissue Regenerative Therapies. Biomaterials 185, 240-275. doi:10.1016/j.biomaterials.2018.09.028

Lu, T., Huang, Y. X., Zhang, C., Chai, M. X., and Zhang, J. (2015). Effect of Pulsed Electromagnetic Field Therapy on the Osteogenic and Adipogenic Differentiation of Bone Marrow Mesenchymal Stem Cells. Genet. Mol. Res. 14, 11535-11542. doi:10.4238/2015.September.28.5

Luo, F., Hou, T., Zhang, Z., Xie, Z., Wu, X., and Xu, J. (2012). Effects of Pulsed Electromagnetic Field Frequencies on the Osteogenic Differentiation of Human Mesenchymal Stem Cells. Orthopedics 35, e526-31. doi:10.3928/0147744720120327-11

Lv, P.-y., Gao, P.-f., Tian, G.-j., Yang, Y.-y., Mo, F.-f., Wang, Z.-h., et al. (2020). Osteocyte-derived Exosomes Induced by Mechanical Strain Promote Human Periodontal Ligament Stem Cell Proliferation and Osteogenic Differentiation via the Mir-181b-5p/pten/akt Signaling Pathway. Stem Cell Res Ther 11, 295. doi:10.1186/s13287-020-01815-3

Ma, B., Liu, F., Li, Z., Duan, J., Kong, Y., Hao, M., et al. (2019). Piezoelectric Nylon11 Nanoparticles with Ultrasound Assistance for High-Efficiency Promotion of Stem Cell Osteogenic Differentiation. J. Mater. Chem. B 7, 1847-1854. doi:10.1039/c8tb03321h

Mancuso, E., Shah, L., Jindal, S., Serenelli, C., Tsikriteas, Z. M., Khanbareh, H., et al. (2021). Additively Manufactured BaTiO3 Composite Scaffolds: A Novel Strategy for Load Bearing Bone Tissue Engineering Applications. Mater. Sci. Eng. C 126, 112192. doi:10.1016/j.msec.2021.112192

McBeath, R., Pirone, D. M., Nelson, C. M., Bhadriraju, K., and Chen, C. S. (2004). Cell Shape, Cytoskeletal Tension, and Rhoa Regulate Stem Cell Lineage
Commitment. Developmental Cel. 6, 483-495. doi:10.1016/s1534-5807(04) 00075-9

McMurray, R. J., Wann, A. K. T., Thompson, C. L., Connelly, J. T., and Knight, M. M. (2013). Surface Topography Regulates Wnt Signaling through Control of Primary Cilia Structure in Mesenchymal Stem Cells. Sci. Rep. 3, 3545. doi:10.1038/srep03545

McNamara, L. E., Sjöström, T., Burgess, K. E. V., Kim, J. J. W., Liu, E., Gordonov, S., et al. (2011). Skeletal Stem Cell Physiology on Functionally Distinct Titania Nanotopographies. Biomaterials 32, 7403-7410. doi:10.1016/ j.biomaterials.2011.06.063

Mitra, D., Whitehead, J., Yasui, O. W., and Leach, J. K. (2017). Bioreactor Culture Duration of Engineered Constructs Influences Bone Formation by Mesenchymal Stem Cells. Biomaterials 146, 29-39. doi:10.1016/ j.biomaterials.2017.08.044

Moser, C., Bardsley, K., El Haj, A. J., Alini, M., Stoddart, M. J., and Bara, J. J. (2018). A Perfusion Culture System for Assessing Bone Marrow Stromal Cell Differentiation on Plga Scaffolds for Bone Repair. Front. Bioeng. Biotechnol. 6, 161. doi:10.3389/fbioe.2018.00161

Murillo, G., Blanquer, A., Vargas-Estevez, C., Barrios, L., Ibáñez, E., Nogués, C., et al. (2017). Electromechanical Nanogenerator-Cell Interaction Modulates Cell Activity. Adv. Mater. 29, 1605048. doi:10.1002/adma.201605048

Nikukar, H., Reid, S., Tsimbouri, P. M., Riehle, M. O., Curtis, A. S. G., and Dalby, M. J. (2013). Osteogenesis of Mesenchymal Stem Cells by Nanoscale Mechanotransduction. ACS nano 7, 2758-2767. doi:10.1021/nn400202j

Niu, H., Lin, D., Tang, W., Ma, Y., Duan, B., Yuan, Y., et al. (2017). Surface Topography Regulates Osteogenic Differentiation of MSCs via Crosstalk between FAK/MAPK and ILK/ $\beta$-Catenin Pathways in a Hierarchically Porous Environment. ACS Biomater. Sci. Eng. 3, 3161-3175. doi:10.1021/ acsbiomaterials.7b00315

Ongaro, A., Pellati, A., Bagheri, L., Fortini, C., Setti, S., and De Mattei, M. (2014). Pulsed Electromagnetic fields Stimulate Osteogenic Differentiation in Human Bone Marrow and Adipose Tissue Derived Mesenchymal Stem Cells. Bioelectromagnetics 35, 426-436. doi:10.1002/bem.21862

Padilla, F., Puts, R., Vico, L., and Raum, K. (2014). Stimulation of Bone Repair with Ultrasound: A Review of the Possible Mechanic Effects. Ultrasonics 54, 1125-1145. doi:10.1016/j.ultras.2014.01.004

Panek, M., Antunović, M., Pribolšan, L., Ivković, A., Gotić, M., Vukasović, A., et al. (2019). Bone Tissue Engineering in a Perfusion Bioreactor Using Dexamethasone-Loaded Peptide Hydrogel. Materials 12, 919. doi:10.3390/ ma12060919

Parmaksiz, M., Lalegül-Ülker, Ö., Vurat, M. T., Elçin, A. E., and Elçin, Y. M. (2021). Magneto-sensitive Decellularized Bone Matrix with or without Low FrequencyPulsed Electromagnetic Field Exposure for the Healing of a Critical-Size Bone Defect. Mater. Sci. Eng. C 124, 112065. doi:10.1016/j.msec.2021.112065

Polley, C., Distler, T., Detsch, R., Lund, H., Springer, A., Boccaccini, A. R., et al. (2020). 3D Printing of Piezoelectric Barium Titanate-Hydroxyapatite Scaffolds with Interconnected Porosity for Bone Tissue Engineering. Materials 13, 1773. doi: $10.3390 / \mathrm{ma} 13071773$

Pongkitwitoon, S., Uzer, G., Rubin, J., and Judex, S. (2016). Cytoskeletal Configuration Modulates Mechanically Induced Changes in Mesenchymal Stem Cell Osteogenesis, Morphology, and Stiffness. Sci. Rep. 6, 34791. doi:10.1038/srep34791

Prè, D., Ceccarelli, G., Visai, L., Benedetti, L., Imbriani, M., Cusella De Angelis, M. G., et al. (20132013). High-frequency Vibration Treatment of Human Bone Marrow Stromal Cells Increases Differentiation toward Bone Tissue. Bone Marrow Res. 2013, 1-13. doi:10.1155/2013/803450

Qian, W., Gong, L., Cui, X., Zhang, Z., Bajpai, A., Liu, C., et al. (2017). Nanotopographic Regulation of Human Mesenchymal Stem Cell Osteogenesis. ACS Appl. Mater. Inter. 9, 41794-41806. doi:10.1021/ acsami.7b16314

Qin, E. C., Ahmed, S. T., Sehgal, P., Vu, V. H., Kong, H., and Leckband, D. E. (2020a). Comparative Effects of N-Cadherin Protein and Peptide Fragments on Mesenchymal Stem Cell Mechanotransduction and Paracrine Function. Biomaterials 239, 119846. doi:10.1016/j.biomaterials.2020.119846

Qin, L., Liu, W., Cao, H., and Xiao, G. (2020b). Molecular Mechanosensors in Osteocytes. Bone Res. 8, 23. doi:10.1038/s41413-020-0099-y

Raftery, R. M., Woods, B., Marques, A. L. P., Moreira-Silva, J., Silva, T. H., Cryan, S.-A., et al. (2016). Multifunctional Biomaterials from the Sea: Assessing the 
Effects of Chitosan Incorporation into Collagen Scaffolds on Mechanical and Biological Functionality. Acta Biomater. 43, 160-169. doi:10.1016/ j.actbio.2016.07.009

Ravichandran, A., Lim, J., Chong, M. S. K., Wen, F., Liu, Y., Pillay, Y. T., et al. (2017). In Vitro cyclic Compressive Loads Potentiate Early Osteogenic Events in Engineered Bone Tissue. J. Biomed. Mater. Res. 105, 2366-2375. doi:10.1002/ jbm.b.33772

Ravichandran, A., Wen, F., Lim, J., Chong, M. S. K., Chan, J. K. Y., and Teoh, S. H. (2018). Biomimetic Fetal Rotation Bioreactor for Engineering Bone TissuesEffect of Cyclic Strains on Upregulation of Osteogenic Gene Expression. J. Tissue Eng. Regen. Med. 12, e2039-e2050. doi:10.1002/term.2635

Ravikumar, K., Boda, S. K., and Basu, B. (2017). Synergy of Substrate Conductivity and Intermittent Electrical Stimulation towards Osteogenic Differentiation of Human Mesenchymal Stem Cells. Bioelectrochemistry 116, 52-64. doi:10.1016/ j.bioelechem.2017.03.004

Reinwald, Y., and El Haj, A. J. (2018). Hydrostatic Pressure in Combination with Topographical Cues Affects the Fate of Bone Marrow-Derived Human Mesenchymal Stem Cells for Bone Tissue Regeneration. J. Biomed. Mater. Res. 106, 629-640. doi:10.1002/jbm.a.36267

Roseti, L., Parisi, V., Petretta, M., Cavallo, C., Desando, G., Bartolotti, I., et al. (2017). Scaffolds for Bone Tissue Engineering: State of the Art and New Perspectives. Mater. Sci. Eng. C 78, 1246-1262. doi:10.1016/j.msec.2017.05.017

Rui, Y. F., Lui, P. P. Y., Ni, M., Chan, L. S., Lee, Y. W., and Chan, K. M. (2011). Mechanical Loading Increased Bmp-2 Expression Which Promoted Osteogenic Differentiation of Tendon-Derived Stem Cells. J. Orthop. Res. 29, 390-396. doi:10.1002/jor.21218

Santos, L. J., Reis, R. L., and Gomes, M. E. (2015). Harnessing Magnetic-Mechano Actuation in Regenerative Medicine and Tissue Engineering. Trends Biotechnology 33, 471-479. doi:10.1016/j.tibtech.2015.06.006

Schreivogel, S., Kuchibhotla, V., Knaus, P., Duda, G. N., and Petersen, A. (2019). Load-induced Osteogenic Differentiation of Mesenchymal Stromal Cells Is Caused by Mechano-regulated Autocrine Signaling. J. Tissue Eng. Regen. Med. 13, 1992-2008. doi:10.1002/term.2948

Schupbach, D., Comeau-Gauthier, M., Harvey, E., and Merle, G. (2020). Wnt Modulation in Bone Healing. Bone 138, 115491. doi:10.1016/ j.bone.2020.115491

Sen, B., Xie, Z., Case, N., Ma, M., Rubin, C., and Rubin, J. (2008). Mechanical Strain Inhibits Adipogenesis in Mesenchymal Stem Cells by Stimulating a Durable $\beta$-Catenin Signal. Endocrinology 149, 6065-6075. doi:10.1210/en.2008-0687

Shah, H. N., Jones, R. E., Borrelli, M. R., Robertson, K., Salhotra, A., Wan, D. C., et al. (2021). Craniofacial and Long Bone Development in the Context of Distraction Osteogenesis. Plast. Reconstr. Surg. 147, 54e-65e. doi:10.1097/ prs.0000000000007451

Shi, Y., Fu, Y., Tong, W., Geng, Y., Lui, P. P. Y., Tang, T., et al. (2012). Uniaxial Mechanical Tension Promoted Osteogenic Differentiation of Rat TendonDerived Stem Cells (Rtdscs) via the Wnt5a-Rhoa Pathway. J. Cell. Biochem. 113, 3133-3142. doi:10.1002/jcb.24190

Silverwood, R. K., Fairhurst, P. G., Sjöström, T., Welsh, F., Sun, Y., Li, G., et al. (2016). Analysis of Osteoclastogenesis/osteoblastogenesis on Nanotopographical Titania Surfaces. Adv. Healthc. Mater. 5, 947-955. doi:10.1002/adhm.201500664

Singh, H., Teoh, S. H., Low, H. T., and Hutmacher, D. W. (2005). Flow Modelling within a Scaffold under the Influence of Uni-Axial and Bi-axial Bioreactor Rotation. J. Biotechnol. 119, 181-196. doi:10.1016/j.jbiotec.2005.03.021

Sittichokechaiwut, A., Edwards, J. H., Edwards, J., Scutt, A., and Reilly, G. (2010). Short Bouts of Mechanical Loading Are as Effective as Dexamethasone at Inducing Matrix Production by Human Bone Marrow Mesenchymal Stem Cells. eCM 20, 45-57. doi:10.22203/ecm.v020a05

Sjöström, T., Dalby, M. J., Hart, A., Tare, R., Oreffo, R. O. C., and Su, B. (2009). Fabrication of Pillar-like Titania Nanostructures on Titanium and Their Interactions with Human Skeletal Stem Cells. Acta Biomater. 5, 1433-1441. doi:10.1016/j.actbio.2009.01.007

Sjöström, T., McNamara, L. E., Meek, R. M. D., Dalby, M. J., and Su, B. (2013). 2d and 3d Nanopatterning of Titanium for Enhancing Osteoinduction of Stem Cells at Implant Surfaces. Adv. Healthc. Mater. 2, 1285-1293. doi:10.1002/ adhm.201200353

Song, F., Jiang, D., Wang, T., Wang, Y., Lou, Y., Zhang, Y., et al. (2017). Mechanical Stress Regulates Osteogenesis and Adipogenesis of Rat Mesenchymal Stem
Cells through PI3K/Akt/GSK-3 $\beta / \beta$-Catenin Signaling Pathway. Biomed. Research International 2017, 1-10. doi:10.1155/2017/6027402

Spangenberg, J., Kilian, D., Czichy, C., Ahlfeld, T., Lode, A., Günther, S., et al. (2021). Bioprinting of Magnetically Deformable Scaffolds. ACS Biomater. Sci. Eng. 7, 648-662. doi:10.1021/acsbiomaterials.0c01371

Stavenschi, E., Corrigan, M. A., Johnson, G. P., Riffault, M., and Hoey, D. A. (2018). Physiological Cyclic Hydrostatic Pressure Induces Osteogenic Lineage Commitment of Human Bone Marrow Stem Cells: A Systematic Study. Stem Cell Res Ther 9, 276. doi:10.1186/s13287-018-1025-8

Steppe, L., Liedert, A., Ignatius, A., and Haffner-Luntzer, M. (2020). Influence of Low-Magnitude High-Frequency Vibration on Bone Cells and Bone Regeneration. Front. Bioeng. Biotechnol. 8, 595139. doi:10.3389/ fbioe.2020.595139

Steward, A. J., and Kelly, D. J. (2015). Mechanical Regulation of Mesenchymal Stem Cell Differentiation. J. Anat. 227, 717-731. doi:10.1111/joa.12243

Stewart, S., Darwood, A., Masouros, S., Higgins, C., and Ramasamy, A. (2020). Mechanotransduction in Osteogenesis. Bone Jt. Res. 9, 1-14. doi:10.1302/20463758.91.Bjr-2019-0043.R2

Sugimoto, A., Miyazaki, A., Kawarabayashi, K., Shono, M., Akazawa, Y., Hasegawa, T., et al. (2017). Piezo Type Mechanosensitive Ion Channel Component 1 Functions as a Regulator of the Cell Fate Determination of Mesenchymal Stem Cells. Sci. Rep. 7, 17696. doi:10.1038/s41598-017-18089-0

Sumanasinghe, R. D., Osborne, J. A., and Loboa, E. G. (2009). Mesenchymal Stem Cell-seeded Collagen Matrices for Bone Repair: Effects of Cyclic Tensile Strain, Cell Density, and media Conditions on Matrix Contraction In Vitro. J. Biomed. Mater. Res. 88A, 778-786. doi:10.1002/jbm.a.31913

Sun, L.-Y., Hsieh, D.-K., Lin, P.-C., Chiu, H.-T., and Chiou, T.-W. (2009). Pulsed Electromagnetic fields Accelerate Proliferation and Osteogenic Gene Expression in Human Bone Marrow Mesenchymal Stem Cells during Osteogenic Differentiation. Bioelectromagnetics 31, a-n. doi:10.1002/ bem. 20550

Sun, L.-Y., Hsieh, D.-K., Yu, T.-C., Chiu, H.-T., Lu, S.-F., Luo, G.-H., et al. (2009). Effect of Pulsed Electromagnetic Field on the Proliferation and Differentiation Potential of Human Bone Marrow Mesenchymal Stem Cells. Bioelectromagnetics 30, 251-260. doi:10.1002/bem.20472

Sun, M., Chi, G., Xu, J., Tan, Y., Xu, J., Lv, S., et al. (2018). Extracellular Matrix Stiffness Controls Osteogenic Differentiation of Mesenchymal Stem Cells Mediated by Integrin a5. Stem Cell Res Ther 9, 52. doi:10.1186/s13287-0180798-0

Tahimic, C. G. T., Long, R. K., Kubota, T., Sun, M. Y., Elalieh, H., Fong, C., et al. (2016). Regulation of Ligand and Shear Stress-Induced Insulin-like Growth Factor 1 (Igf1) Signaling by the Integrin Pathway. J. Biol. Chem. 291, 8140-8149. doi:10.1074/jbc.M115.693598

Tan, S. D., de Vries, T. J., Kuijpers-Jagtman, A. M., Semeins, C. M., Everts, V., and Klein-Nulend, J. (2007). Osteocytes Subjected to Fluid Flow Inhibit Osteoclast Formation and Bone Resorption. Bone 41, 745-751. doi:10.1016/ j.bone.2007.07.019

Tang, Y., Wu, C., Wu, Z., Hu, L., Zhang, W., and Zhao, K. (2017). Fabrication and In Vitro Biological Properties of Piezoelectric Bioceramics for Bone Regeneration. Sci. Rep. 7, 43360. doi:10.1038/srep43360

Thompson, W. R., Guilluy, C., Xie, Z., Sen, B., Brobst, K. E., Yen, S. S., et al. (2013). Mechanically Activated Fyn Utilizes Mtorc2 to Regulate Rhoa and Adipogenesis in Mesenchymal Stem Cells. Stem Cells 31, 2528-2537. doi:10.1002/stem.1476

Thompson, W. R., Rubin, C. T., and Rubin, J. (2012). Mechanical Regulation of Signaling Pathways in Bone. Gene 503, 179-193. doi:10.1016/ j.gene.2012.04.076

Thompson, W. R., Yen, S. S., Uzer, G., Xie, Z., Sen, B., Styner, M., et al. (2018). Larg Gef and Arhgap18 Orchestrate Rhoa Activity to Control Mesenchymal Stem Cell Lineage. Bone 107, 172-180. doi:10.1016/j.bone.2017.12.001

Thrivikraman, G., Boda, S. K., and Basu, B. (2018). Unraveling the Mechanistic Effects of Electric Field Stimulation towards Directing Stem Cell Fate and Function: A Tissue Engineering Perspective. Biomaterials 150, 60-86. doi:10.1016/j.biomaterials.2017.10.003

Tsai, M.-T., Li, W.-J., Tuan, R. S., and Chang, W. H. (2009). Modulation of Osteogenesis in Human Mesenchymal Stem Cells by Specific Pulsed Electromagnetic Field Stimulation. J. Orthop. Res. 27, 1169-1174. doi:10.1002/jor.20862 
Tsimbouri, P. M., Childs, P. G., Pemberton, G. D., Yang, J., Jayawarna, V., Orapiriyakul, W., et al. (2017). Stimulation of 3d Osteogenesis by Mesenchymal Stem Cells Using a Nanovibrational Bioreactor. Nat. Biomed. Eng. 1, 758-770. doi:10.1038/s41551-017-0127-4

Tyrovola, J. B., and Odont, X. (2015). The "mechanostat Theory" of Frost and the Opg/rankl/rank System. J. Cell. Biochem. 116, 2724-2729. doi:10.1002/ jcb. 25265

Wang, J., Tang, N., Xiao, Q., Zhao, L., Li, Y., Li, J., et al. (2016a). The Potential Application of Pulsed Ultrasound on Bone Defect Repair via Developmental Engineering: An In Vitro Study. Artif. organs 40, 505-513. doi:10.1111/aor.12578

Wang, J., Wang, C. D., Zhang, N., Tong, W. X., Zhang, Y. F., Shan, S. Z., et al. (2016b). Mechanical Stimulation Orchestrates the Osteogenic Differentiation of Human Bone Marrow Stromal Cells by Regulating Hdac1. Cell Death Dis 7-e2221. doi:10.1038/cddis.2016.112

Wang, M., Favi, P., Cheng, X., Golshan, N. H., Ziemer, K. S., Keidar, M., et al. (2016c). Cold Atmospheric Plasma (Cap) Surface Nanomodified 3d Printed Polylactic Acid (Pla) Scaffolds for Bone Regeneration. Acta Biomater. 46, 256-265. doi:10.1016/j.actbio.2016.09.030

Wang, N., Tytell, J. D., and Ingber, D. E. (2009). Mechanotransduction at a Distance: Mechanically Coupling the Extracellular Matrix with the Nucleus. Nat. Rev. Mol. Cell Biol 10, 75-82. doi:10.1038/nrm2594

Wang, T., Yang, L., Jiang, J., Liu, Y., Fan, Z., Zhong, C., et al. (2019). Pulsed Electromagnetic fields: Promising Treatment for Osteoporosis. Osteoporos. Int. 30, 267-276. doi:10.1007/s00198-018-04822-6

Wechsler, M. E., Hermann, B. P., and Bizios, R. (2016). Adult Human Mesenchymal Stem Cell Differentiation at the Cell Population and SingleCell Levels under Alternating Electric Current. Tissue Eng. C: Methods 22, 155-164. doi:10.1089/ten.TEC.2015.0324

Wojda, S. J., and Donahue, S. W. (2018). Parathyroid Hormone for Bone Regeneration. J. Orthop. Res. 36, 2586-2594. doi:10.1002/jor.24075

W. Sawyer, S., Zhang, K., Zhang, K., A. Horton, J., and Soman, P. (2020). Perfusionbased Co-culture Model System for Bone Tissue Engineering. AIMS Bioeng. 7, 91-105. doi:10.3934/bioeng.2020009

Wu, J., Chen, T., Wang, Z., Chen, X., Qu, S., Weng, J., et al. (2020). Joint Construction of Micro-vibration Stimulation and Bcp Scaffolds for Enhanced Bioactivity and Self-Adaptability Tissue Engineered Bone Grafts. J. Mater. Chem. B 8, 4278-4288. doi:10.1039/d0tb00223b

Wu, J., Zhao, J., Sun, L., Pan, Y., Wang, H., and Zhang, W.-B. (2018). Long Noncoding Rna H19 Mediates Mechanical Tension-Induced Osteogenesis of Bone Marrow Mesenchymal Stem Cells via Fak by Sponging Mir-138. Bone 108, 62-70. doi:10.1016/j.bone.2017.12.013

Wu, W., Le, A. V., Mendez, J. J., Chang, J., Niklason, L. E., and Steinbacher, D. M. (2015). Osteogenic Performance of Donor-Matched Human Adipose and Bone Marrow Mesenchymal Cells under Dynamic Culture. Tissue Eng. Part A 21, 1621-1632. doi:10.1089/ten.TEA.2014.0115

Wu, X., Li, Y., Cao, Z., Xie, Y., Fu, C., and Chen, H. (2021). Mechanism of Cyclic Tensile Stress in Osteogenic Differentiation of Human Periodontal Ligament Stem Cells. Calcif Tissue Int. 108, 640-653. doi:10.1007/s00223-020-00789-x

Xiao, F., Zuo, B., Tao, B., Wang, C., Li, Y., Peng, J., et al. (2021). Exosomes Derived from Cyclic Mechanical Stretch-Exposed Bone Marrow Mesenchymal Stem Cells Inhibit RANKL-Induced Osteoclastogenesis through the NF- $\mathrm{kB}$ Signaling Pathway. Ann. Transl Med. 9, 798. doi:10.21037/atm-21-1838

Yan, H., Liu, X., Zhu, M., Luo, G., Sun, T., Peng, Q., et al. (2016). Hybrid Use of Combined and Sequential Delivery of Growth Factors and Ultrasound Stimulation in Porous Multilayer Composite Scaffolds to Promote Both Vascularization and Bone Formation in Bone Tissue Engineering. J. Biomed. Mater. Res. 104, 195-208. doi:10.1002/jbm.a.35556

Yang, L., Huang, J., Yang, S., Cui, W., Wang, J., Zhang, Y., et al. (2018). Bone Regeneration Induced by Local Delivery of a Modified Pth-Derived Peptide from Nanohydroxyapatite/chitosan Coated True Bone Ceramics. ACS Biomater. Sci. Eng. 4, 3246-3258. doi:10.1021/acsbiomaterials.7b00780

Ye, L., Xu, J., Mi, J., He, X., Pan, Q., Zheng, L., et al. (2021). Biodegradable Magnesium Combined with Distraction Osteogenesis Synergistically Stimulates Bone Tissue Regeneration via Cgrp-Fak-Vegf Signaling axis. Biomaterials 275, 120984. doi:10.1016/j.biomaterials.2021.120984

Yong, K. W., Choi, J. R., Choi, J. Y., and Cowie, A. C. (2020). Recent Advances in Mechanically Loaded Human Mesenchymal Stem Cells for Bone Tissue Engineering. Ijms 21, 5816. doi:10.3390/ijms21165816
You, L., Temiyasathit, S., Lee, P., Kim, C. H., Tummala, P., Yao, W., et al. (2008). Osteocytes as Mechanosensors in the Inhibition of Bone Resorption Due to Mechanical Loading. Bone 42, 172-179. doi:10.1016/j.bone.2007.09.047

Yourek, G., McCormick, S. M., Mao, J. J., and Reilly, G. C. (2010). Shear Stress Induces Osteogenic Differentiation of Human Mesenchymal Stem Cells. Regenerative Med. 5, 713-724. doi:10.2217/rme.10.60

Yu, H.-S., Won, J.-E., Jin, G.-Z., and Kim, H.-W. (2012). Construction of Mesenchymal Stem Cell-Containing Collagen Gel with a Macrochanneled Polycaprolactone Scaffold and the Flow Perfusion Culturing for Bone Tissue Engineering. BioResearch open access 1, 124-136. doi:10.1089/biores.2012.0234

Yu, P., Ning, C., Zhang, Y., Tan, G., Lin, Z., Liu, S., et al. (2017). Bone-inspired Spatially Specific Piezoelectricity Induces Bone Regeneration. Theranostics 7, 3387-3397. doi:10.7150/thno.19748

Yuan, H., Zhou, Y., Lee, M.-S., Zhang, Y., and Li, W.-J. (2016). A Newly Identified Mechanism Involved in Regulation of Human Mesenchymal Stem Cells by Fibrous Substrate Stiffness. Acta Biomater. 42, 247-257. doi:10.1016/ j.actbio.2016.06.034

Yue, Y., Yang, X., Wei, X., Chen, J., Fu, N., Fu, Y., et al. (2013). Osteogenic Differentiation of Adipose-derived Stem Cells Prompted by Low-intensity Pulsed Ultrasound. Cell Prolif. 46, 320-327. doi:10.1111/cpr.12035

Zhang, C., Liu, W., Cao, C., Zhang, F., Tang, Q., Ma, S., et al. (2018). Modulating Surface Potential by Controlling the $\beta$ Phase Content in Poly(vinylidene Fluoridetrifluoroethylene) Membranes Enhances Bone Regeneration. Adv. Healthc. Mater. 7, 1701466. doi:10.1002/adhm.201701466

Zhang, C., Lu, Y., Zhang, L., Liu, Y., Zhou, Y., Chen, Y., et al. (2015). Influence of Different Intensities of Vibration on Proliferation and Differentiation of Human Periodontal Ligament Stem Cells. aoms 3, 638-646. doi:10.5114/ aoms.2015.52370

Zhang, J., He, X., Chen, X., Wu, Y., Dong, L., Cheng, K., et al. (2020). Enhancing Osteogenic Differentiation of Bmscs on High Magnetoelectric Response Films. Mater. Sci. Eng. C 113, 110970. doi:10.1016/j.msec.2020.110970

Zhang, N., Chow, S. K.-H., Leung, K.-S., and Cheung, W.-H. (2017). Ultrasound as a Stimulus for Musculoskeletal Disorders. J. orthopaedic translation 9, 52-59. doi:10.1016/j.jot.2017.03.004

Zhang, S., Yang, Q., Zhao, W., Qiao, B., Cui, H., Fan, J., et al. (2016). In Vitro and In Vivo Biocompatibility and Osteogenesis of Graphene-Reinforced Nanohydroxyapatite Polyamide66 Ternary Biocomposite as Orthopedic Implant Material. Ijn 11, 3179-3189. doi:10.2147/ijn.S105794

Zhang, Z.-Y., Teoh, S.-H., Chong, M. S. K., Lee, E. S. M., Tan, L.-G., Mattar, C. N., et al. (2010a). Neo-vascularization and Bone Formation Mediated by Fetal Mesenchymal Stem Cell Tissue-Engineered Bone Grafts in Critical-Size Femoral Defects. Biomaterials 31, 608-620. doi:10.1016/j.biomaterials.2009.09.078

Zhang, Z.-Y., Teoh, S. H., Chong, W.-S., Foo, T.-T., Chng, Y.-C., Choolani, M., et al. (2009). A Biaxial Rotating Bioreactor for the Culture of Fetal Mesenchymal Stem Cells for Bone Tissue Engineering. Biomaterials 30, 2694-2704. doi:10.1016/j.biomaterials.2009.01.028

Zhang, Z.-Y., Teoh, S. H., Teo, E. Y., Khoon Chong, M. S., Shin, C. W., Tien, F. T., et al. (2010b). A Comparison of Bioreactors for Culture of Fetal Mesenchymal Stem Cells for Bone Tissue Engineering. Biomaterials 31, 8684-8695. doi:10.1016/j.biomaterials.2010.07.097

Zhao, L., Weir, M. D., and Xu, H. H. K. (2010). An Injectable Calcium PhosphateAlginate Hydrogel-Umbilical Cord Mesenchymal Stem Cell Paste for Bone Tissue Engineering. Biomaterials 31, 6502-6510. doi:10.1016/ j.biomaterials.2010.05.017

Zhao, Y.-H., Lv, X., Liu, Y.-L., Zhao, Y., Li, Q., Chen, Y.-J., et al. (2015). Hydrostatic Pressure Promotes the Proliferation and Osteogenic/chondrogenic Differentiation of Mesenchymal Stem Cells: The Roles of Rhoa and Rac1. Stem Cel. Res. 14, 283-296. doi:10.1016/j.scr.2015.02.006

Zhao, Y., Huang, Y., Jia, L., Wang, R., Tan, K., and Li, W. (2021). A Novel Tension Machine Promotes Bone Marrow Mesenchymal Stem Cell Osteoblastic and Fibroblastic Differentiation by Applying Cyclic Tension. Stem Cell Int. 2021, 1-15. doi:10.1155/2021/6647651

Zhou, J., Li, B., Han, Y., and Zhao, L. (2016a). The Osteogenic Capacity of Biomimetic Hierarchical Micropore/nanorod-Patterned Sr-Ha Coatings with Different Interrod Spacings. Nanomedicine: Nanotechnology, Biol. Med. 12, 1161-1173. doi:10.1016/j.nano.2016.01.011

Zhou, X., Castro, N. J., Zhu, W., Cui, H., Aliabouzar, M., Sarkar, K., et al. (2016b). Improved Human Bone Marrow Mesenchymal Stem Cell Osteogenesis in 3d 
Bioprinted Tissue Scaffolds with Low Intensity Pulsed Ultrasound Stimulation. Sci. Rep. 6, 32876. doi:10.1038/srep32876

Zhu, G., Zeng, C., Qian, Y., Yuan, S., Ye, Z., Zhao, S., et al. (2021). Tensile Strain Promotes Osteogenic Differentiation of Bone Marrow Mesenchymal Stem Cells through Upregulating Lncrna-Meg3. Histology and histopathology, 18365. doi:10.14670/hh-18-365

Zura, R., Xiong, Z., Einhorn, T., Watson, J. T., Ostrum, R. F., Prayson, M. J., et al. (2016). Epidemiology of Fracture Nonunion in 18 Human Bones. JAMA Surg. 151, e162775. doi:10.1001/jamasurg.2016.2775

Conflict of Interest: The authors declare that the research was conducted in the absence of any commercial or financial relationships that could be construed as a potential conflict of interest.
Publisher's Note: All claims expressed in this article are solely those of the authors and do not necessarily represent those of their affiliated organizations, or those of the publisher, the editors and the reviewers. Any product that may be evaluated in this article, or claim that may be made by its manufacturer, is not guaranteed or endorsed by the publisher.

Copyright $\odot 2021 \mathrm{Hao}, \mathrm{Xu}$, Wang, Wang, Li, Chen, Hu, Chen, Huang, Chen and $\mathrm{Li}$. This is an open-access article distributed under the terms of the Creative Commons Attribution License (CC BY). The use, distribution or reproduction in other forums is permitted, provided the original author(s) and the copyright owner(s) are credited and that the original publication in this journal is cited, in accordance with accepted academic practice. No use, distribution or reproduction is permitted which does not comply with these terms. 